\title{
ANALYSIS OF THE RESPIRATORY RESPONSE TO CARBON DIOXIDE INHALATION IN VARYING CLINICAL STATES OF HYPERCAPNIA, ANOXIA, AND ACID-BASE DERANGEMENT
}

\author{
BY JAMES K. ALEXANDER,1, 2, 3 JOHN R. WEST, † JOHN A. WOOD, AND
} DICKINSON W. RICHARDS

\author{
(From the Department of Medicine, Columbia University College of Physicians and Surgeons, \\ and the Cardio-pulmonary Laboratory of the Presbyterian Hospital, \\ New York City, N. Y.)
}

(Submitted for publication July 30, 1954 ; accepted December 1, 1954)

Individuals with cor pulmonale secondary to chronic pulmonary emphysema tend to have pulmonary ventilation which is less than normal both at rest and during exercise, despite the presence of factors ordinarily making for increased ventilation such as anoxemia and acidosis (1), and despite the fact that these ventilatory levels may be appreciably less than the observed maximum breathing capacity. Moreover, the ventilatory response to increased $\mathrm{CO}_{2}$ in the inspired air may be less than normal in certain patients with pulmonary emphysema (2-5). These observations have been interpreted as indicating a disorder of the chemical regulation of respiration associated with emphysema.

The present study was undertaken to define more specifically the nature of this disorder in terms of the sensitivity of the neural regulatory mechanism to the carbon dioxide-hydrogen ion stimulus, and to characterize the conditions under which such a disorder might exist. Sensitivity of the neural regulatory mechanism to $\mathrm{CO}_{2}$ and/or hydrogen ion has been evaluated quantitatively by correlation of arterial blood hydrogen ion concentration and $\mathrm{CO}_{2}$ tension with effective alveolar ventilation when changes are brought about by varying amounts of carbon dioxide in the inspired air.

In the present investigation, the effects of $\mathrm{CO}_{2}$ inhalation in normal subjects have been compared

\footnotetext{
1 This work was done partly during the tenure of a Research Fellowship of the American Heart Association.

2 Present Address: Department of Medicine, Baylor University College of Medicine, Houston, Texas.

supported in part by a gift from the Charles A. Frueauff Foundation.

† Dr. West died June 29, 1954.
}

with those in emphysematous subjects having disease of varying severity.

In order to evaluate separately the possible effects of chronic anoxemia, chronic acidosis, and chronic hypercapnia in conditioning the response of patients with advanced pulmonary emphysema, patients without pulmonary disease who exhibited such derangements have also been studied. For investigation of the effects of chronic anoxemia, patients with cyanotic congenital heart disease were chosen; for the effects of chronic acidosis patients with long standing renal failure and nitrogen retention; and for the effects of hypercapnia patients with chronic metabolic alkalosis secondary to hyperadrenalism.

\section{MATERIALS AND METHODS}

Material for study comprised twelve normal subjects, thirteen patients with chronic pulmonary emphysema, three patients with cyanotic congenital heart disease, three patients with chronic renal disease and uremia, and two patients with Cushing's syndrome and chronic metabolic alkalosis.

Subjects were studied in the post-absorptive state. Observations at rest and during a standard exercise test were carried out as outlined by Baldwin, Cournand, and Richards (6). To determine sensitivity to the carbon dioxide-hydrogen ion stimulus, three sets of observations were made on each subject, allowing the determination of three points on the stimulus response curve. In each instance the subject breathed in succession room air, 3 per cent $\mathrm{CO}_{2}$ in air, and 5 per cent $\mathrm{CO}_{2}$ in air through a two-way low resistance respiratory valve with a dead space of $60 \mathrm{ml}$. The $\mathrm{CO}_{2}$ mixture was supplied continuously from a tank through an anesthesia bag which was in turn connected to the inspiratory side of the respiratory valve. As previously reported by Nielsen (7), it was found that for both normal subjects and patients approximately twenty to twenty-five minutes' inhalation of the $\mathrm{CO}_{2}$ gas mixtures used was required to achieve a steady state in terms of pulmonary ventilation, 
respiratory frequency, and respiratory exchange ratio. Therefore observations were made only after twenty to thirty-five minutes of continuous inhalation of $\mathrm{CO}_{2}$. Expired air was collected for a three-minute period, and a steadily and continuously drawn sample of arterial blood was obtained during the middle minute from an indwelling needle in a brachial artery.

If it is assumed that in a steady state there is a consistent relationship between the arterial blood concentrations and those intra- or extracellular concentrations of certain chemical agents acting at receptor and integrative levels on the nervous system, simultaneous measurement of blood concentrations and pulmonary ventilation permits estimation of the sensitivity of the respiratory nervous regulatory mechanism as a whole to these stimuli. The values relating effective alveolar ventilation, either in terms of ventilation ratio (VR) or alveolar ventilation per square meter body surface area $\left(\mathrm{VA} / \mathrm{M}^{2}\right)$, to changes in arterial $\mathrm{CO}_{2}$ tension $\left(\mathrm{PaCO}_{2}\right)$ and hydrogen ion concentration $\left(\mathrm{H}^{+}\right)$a were obtained from graphs in which VR and $\mathrm{VA} / \mathrm{M}^{2}$ were plotted against $\mathrm{PaCO}_{2}$ and $\left(\mathrm{H}^{+}\right)$a, respectively. For both $\mathrm{PaCO}_{2}$ and $\left(\mathrm{H}^{+}\right)$a the relationship was found to be essentially linear, so that a straight line best fitting the three points could be drawn. The slopes of the lines so constructed have been utilized to determine the changes in arterial $\mathrm{CO}_{2}$ tension or hydrogen ion concentration required to double the effective alveolar ventilation $\left(\mathrm{PaCO}_{2} / \mathrm{VR},\left(\mathrm{H}^{+}\right) \mathrm{a} / \mathrm{VR}\right)$ and to determine the increase in effective alveolar ventilation per square meter body surface area associated with unit rise in arterial $\mathrm{CO}_{2}$ tension or hydrogen ion concentration $\left(\nabla A / \mathrm{M}^{2} / \mathrm{PaCO}_{2}, \nabla A / \mathrm{M}^{2} /\left(\mathrm{H}^{+}\right) \mathrm{a}\right)$. To arrive at a figure representing the change in arterial $\mathrm{CO}_{2}$ tension or hydrogen ion concentration necessary to double the effective alveolar ventilation when the latter did not actually occur, the observed slope was extrapolated.

Oxygen consumption and respiratory exchange ratios were calculated in the usual way from expired air analyses. Physiological dead space ventilation was obtained using the Bohr relationship for carbon dioxide (8) assuming the arterial $\mathrm{CO}_{2}$ tension to be equivalent to the mean alveolar $\mathrm{CO}_{2}$ tension $(9,10)$. Effective alveolar ventilation was taken as the difference between total pulmonary ventilation and dead space ventilation. Mean alveolar oxygen tensions were calculated from the usual "alveolar" equation when the inspired gas mixture was room air, and from a generalized form of the same relationships when carbon dioxide was added to the inspired air.4 Arterial whole blood buffer base was cal-

4 The general form of the alveolar equation may be readily derived by expressing the alveolar respiratory exchange ratio in terms of fractions of oxygen and $\mathrm{CO}_{2}$ in alveolar and inspired air, converting certain of these dry fractions to gas tension, and solving the expression for alveolar oxygen tension $(11,12)$. The form used in the present study was as follows:

$\mathrm{PAO}_{2}=$

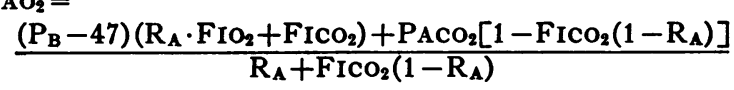

TABLE I

Symbols

B.S.A. Body Surface Area, square meters.

$\mathbf{R}_{\mathbf{E}} \quad$ Respiratory exchange ratio $(R Q)$, expired air.

$R_{A} \quad$ Respiratory exchange ratio (RQ), alveolar air.

$\dot{\mathrm{V}}_{2}$ Oxygen consumption, ml. per min. STPD (Standard temperature and pressure, dry).

$M^{2} \quad$ Square meters of body surface area.

$\dot{\mathbf{V}}_{\mathbf{E}} \quad$ Total ventilation (expired air), liters per minute, BTPS (Body temperature and pressure, saturated with water vapor).

$V_{D} \quad$ Ventilatory dead space total, i.e., instrumental plus personal, ml. BTPS.

$\mathrm{PAO}_{2} \quad$ "Effective" alveolar oxygen tension, $\mathrm{mm} . \mathrm{Hg}$

$\dot{\mathrm{V}}_{\mathbf{A}} \quad$ Effective alveolar ventilation, liters per $\mathrm{min}$. BTPS.

VR Alveolar ventilation ratio, i.e., observed effective alveolar ventilation divided by resting effective alveolar ventilation.

pHs Arterial blood pH (serum).

$\left(\mathrm{H}^{+}\right)$a Hydrogen ion concentration, arterial blood, in billionths of moles per liter.

$\mathrm{Csco}_{2}$ Serum carbon dioxide content, arterial blood, volumes per cent.

$\mathrm{PacO}_{2}$ Carbon dioxide tension, arterial blood, $\mathrm{mm} . \mathrm{Hg}$

$\mathrm{SaO}_{2}$ Oxygen saturation, arterial blood, per cent.

$\mathrm{V}_{\mathrm{c}} \quad$ Hematocrit, arterial blood.

$\left(B_{B_{+}}\right)$b Buffer base, whole blood, mEq. per liter.

$f$ Respiratory frequency as breaths per minute.

VC Vital capacity, ml. BTPS.

$\mathrm{V}_{\mathrm{T}}$ Tidal volume, $\mathrm{ml}$. BTPS.

FRC Functional residual capacity, liters BTPS.

RV Residual volume to total capacity ratio.

MBC Maximum breathing capacity, liters per min. BTPS.

$\mathrm{PacO}_{2}$ Change in arterial $\mathrm{CO}_{2}$ tension, $\mathrm{mm}$. $\mathrm{Hg}$, necesVR sary to double the effective alveolar ventilation.

$\dot{\mathrm{V}}_{\mathrm{A}} / \mathrm{M}^{\mathbf{2}}$ Increase in effective alveolar ventilation, liters

$\mathrm{PaCO}_{2}$ per minute BTPS per sq. meter BSA, associated with a rise of $1 \mathrm{~mm} . \mathrm{Hg}$ in arterial $\mathrm{CO}_{2}$ tension.

$\left(\mathrm{H}^{+}\right) \mathrm{a}$ Change in arterial hydrogen ion concentration, VR billionths of moles per liter, necessary to double the effective alveolar ventilation.

$\dot{V}_{A} / M^{2}$ Increase in effective alveolar ventilation, liters

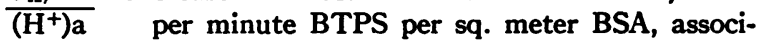
ated with a rise in arterial hydrogen ion concentration of 1 billionth of a mole per liter.

culated from the Singer-Hastings nomogram (13). A list of the symbols used (14) in this report appears in Table I.

Total pulmonary ventilation per unit time and respiratory frequency were measured with a Tissot gasometer and suitable recording apparatus. Fractions of $\mathrm{CO}_{2}$ and

This reduces to the usual alveolar equation in the special case where $\mathrm{CO}_{2}$ in the inspired air is negligible. $\mathrm{R}_{\Delta}$ was taken as equivalent to $R_{\mathbf{m}}$. 
oxygen in inspired and expired air were determined by the Scholander technique (15). Arterial blood oxygen content, $\mathrm{CO}_{2}$ content, and oxygen capacity were determined on the Van Slyke manometric apparatus. Arterial blood $\mathrm{pH}$ was measured with a Cambridge glass electrode $\mathrm{pH}$ meter at $38^{\circ} \mathrm{C}$. within ten minutes of removal from the vessel, transfer of blood from artery to glass electrode being accomplished without exposure to air. Arterial serum $\mathrm{CO}_{2}$ content was calculated from the diagram of Van Slyke and $\mathrm{CO}_{2}$ tension from the Henderson-Hasselbalch relationship. Lung volumes and maximum breathing capacity were measured with a Benedict-Roth respirometer. The open circuit method was used for determining functional residual capacity (16).

\section{RESULTS}

\section{Normal group}

The data obtained from observations on twelve normal subjects appear in Tables II-V, with the spirometric and ventilatory data in Tables II and III. Respiratory exchange ratios showed only small variation, there being an average change of 0.06 from the resting value with inhalation of 3 per cent $\mathrm{CO}_{2}$ and 0.05 with 5 per cent $\mathrm{CO}_{2}$. Respiratory frequency tended to increase only slightly with $\mathrm{CO}_{2}$ inhalation, usually not more than about five breaths per minute. In two instances the rate was slower with 5 per cent $\mathrm{CO}_{2}$ inhalation than with 3 per cent $\mathrm{CO}_{2}$. A greater tidal volume largely accounted for the increased pulmonary ventilation with $\mathrm{CO}_{2}$ inhalation, rising to 158 per cent of the resting tidal volume on 3 per cent $\mathrm{CO}_{2}$, and to 232 per cent on 5 per cent $\mathrm{CO}_{2}$. Oxygen consumption per square meter body surface area showed no consistent or marked change with 3 per cent $\mathrm{CO}_{2}$ inhalation, and only an inconstant tendency to rise with 5 per cent $\mathrm{CO}_{2}$ (average increase $11 \mathrm{ml}$.). Average total pulmonary ventilation rose to 202 per cent of the resting value with 3 per cent $\mathrm{CO}_{2}$ inhalation, and to 329 per cent with 5 per cent $\mathrm{CO}_{2}$. In all instances but two, the physiological dead space increased appreciably with 5 per cent $\mathrm{CO}_{2}$ inhalation. If the three instances in which a large dead space flutter valve was used are excluded, and the personal dead space is determined by deducting the instrumental dead space $(60 \mathrm{ml}$.) from the observed total dead space $\left(V_{D}\right)$, then there was an average increase in the personal physiological dead space of $39 \mathrm{ml}$. or 47 per cent with 3 per cent $\mathrm{CO}_{2}$ inhalation, and of $75 \mathrm{ml}$. or 61 per cent with 5 per cent $\mathrm{CO}_{2}$. The average personal physiological dead space was $92 \mathrm{ml}$. The average values for calculated mean alveolar oxygen tension were 97 $\mathrm{mm}$. $\mathrm{Hg}$ at rest, $117 \mathrm{~mm}$. $\mathrm{Hg}$ with 3 per cent $\mathrm{CO}_{2}$ inhalation, and $125 \mathrm{~mm}$. $\mathrm{Hg}$ with 5 per cent $\mathrm{CO}_{2}$. An increase of 3 per cent and 5 per cent $\mathrm{CO}_{2}$ occurred in each instance, with little individual variation from the average figures. Average effective alveolar ventilation rose to 222 per cent of the resting value with 3 per cent $\mathrm{CO}_{2}$ inhalation, and to 382 per cent with 5 per cent $\mathrm{CO}_{2}$.

Table IV contains the data from arterial blood analyses. The $\mathrm{pH}$ values are also expressed in terms of absolute hydrogen ion concentration as billionths of moles per liter ${ }^{5}$ in order that the

5 Hydrogen ion concentration as billionths of moles per liter is related to $\mathrm{pH}$ in the following way: $\left(\mathrm{H}^{+}\right)=$anti$\log (9-\mathrm{pH})$.

TABLE II

Spirometric data in twelve normal subjects *

\begin{tabular}{|c|c|c|c|c|c|c|c|c|}
\hline \multirow[b]{2}{*}{ Subject } & \multirow[b]{2}{*}{ Sex } & \multirow[b]{2}{*}{ Age } & \multirow[b]{2}{*}{ B.S.A. } & \multicolumn{2}{|c|}{ Vital capacity } & \multicolumn{3}{|c|}{ Maximum breathing capacity } \\
\hline & & & & $m l$. & $\%$ pred. & L./min. & $L . / \min . / M^{2}$ & $\%$ pred. \\
\hline $\begin{array}{l}\text { T. J. } \\
\text { J. R. W. } \\
\text { J. A. W. } \\
\text { J. K. A. } \\
\text { H. J. } \\
\text { J. D. } \\
\text { H. M. } \\
\text { P. P. } \\
\text { V. M. } \\
\text { N. B. } \\
\text { B. O. } \\
\text { M. B. }\end{array}$ & $\begin{array}{l}\mathbf{F} \\
\mathbf{M} \\
\mathbf{M} \\
\mathbf{M} \\
\mathbf{M} \\
\mathbf{M} \\
\mathbf{M} \\
\mathbf{M} \\
\mathbf{M} \\
\mathbf{F} \\
\mathbf{M} \\
\mathbf{F}\end{array}$ & $\begin{array}{l}43 \\
35 \\
30 \\
32 \\
17 \\
37 \\
35 \\
35 \\
18 \\
27 \\
32 \\
24\end{array}$ & $\begin{array}{l}1.99 \\
1.91 \\
1.96 \\
2.10 \\
1.89 \\
1.73 \\
1.79 \\
1.68 \\
1.67 \\
1.70 \\
1.76 \\
1.52\end{array}$ & $\begin{array}{l}3,340 \\
4,200 \\
5,380 \\
7,050 \\
4,670 \\
4,685 \\
5,030 \\
4,130 \\
4,360 \\
3,610 \\
4,530 \\
3,050\end{array}$ & $\begin{array}{l}100 \\
100 \\
100 \\
100 \\
100 \\
100 \\
100 \\
100 \\
100 \\
100 \\
100 \\
100\end{array}$ & $\begin{array}{r}78 \\
144 \\
174 \\
182 \\
106 \\
107 \\
120 \\
121 \\
137 \\
151 \\
122 \\
108\end{array}$ & $\begin{array}{l}39 \\
75.4 \\
89 \\
87 \\
56 \\
62 \\
67 \\
71.9 \\
82.2 \\
89 \\
69.5 \\
72\end{array}$ & $\begin{array}{r}78 \\
100 \\
100 \\
100 \\
74 \\
93 \\
100 \\
100 \\
100 \\
100 \\
100 \\
100\end{array}$ \\
\hline
\end{tabular}

* A value of 100 for per cent predicted vital or maximum breathing capacity indicates that the observed value was 100 per cent or above. 
TABLE III

Physiologic data relative to pulmonary ventilation at rest and during $\mathrm{CO}_{2}$ inhalation in twolve normal swbjects

\begin{tabular}{|c|c|c|c|c|c|c|c|c|c|c|}
\hline \multirow[b]{2}{*}{ Subject } & \multirow{2}{*}{$\begin{array}{l}\text { Inspired } \\
\text { gas } \\
\text { mixture }\end{array}$} & \multirow{2}{*}{$\underset{\text { mixture }}{\text { Timine }}$} & \multirow[b]{2}{*}{ f } & \multirow[b]{2}{*}{$\mathbf{R} \mathbf{z}$} & \multirow[b]{2}{*}{$\dot{V}_{O_{2} / M^{2}}$} & \multirow[b]{2}{*}{$\mathbf{V}_{\mathbf{T}}$} & \multirow[b]{2}{*}{$\dot{\mathbf{V}} \mathbf{z}$. } & \multicolumn{2}{|c|}{$V_{D}$} & \multirow{2}{*}{$\begin{array}{l}\text { Calc. } \\
\mathbf{P}_{\mathbf{A}_{0}}\end{array}$} \\
\hline & & & & & & & & $m l$. & $\overline{\%}$ & \\
\hline T. J. & $\begin{array}{l}\text { Room air } \\
3 \% \mathrm{CO}_{2} \\
5 \% \mathrm{CO}_{2}\end{array}$ & $\begin{array}{l}\overline{20} \\
24\end{array}$ & $\begin{array}{l}17.7 \\
31 \\
33.3\end{array}$ & $\begin{array}{l}0.70 \\
0.72 \\
0.72\end{array}$ & $\begin{array}{l}125 \\
147 \\
165\end{array}$ & $\begin{array}{l}381 \\
487 \\
891\end{array}$ & $\begin{array}{r}6.76 \\
15.10 \\
29.65\end{array}$ & $\begin{array}{l}163 \\
183 \\
321\end{array}$ & $\begin{array}{l}42.8 \\
37.7 \\
36\end{array}$ & $\begin{array}{r}96 \\
117 \\
128\end{array}$ \\
\hline J. R. W.* & $\begin{array}{l}\text { Room air } \\
3 \% \mathrm{CO}_{2} \\
5 \% \mathrm{CO}_{2}\end{array}$ & $\begin{array}{l}\overline{26} \\
21\end{array}$ & $\begin{array}{l}8.7 \\
12.4 \\
14\end{array}$ & $\begin{array}{l}0.77 \\
0.87 \\
0.82\end{array}$ & $\begin{array}{l}123 \\
130 \\
133\end{array}$ & $\begin{array}{r}592 \\
982 \\
1,377\end{array}$ & $\begin{array}{r}5.16 \\
12.16 \\
19.26\end{array}$ & $\begin{array}{l}192 \\
382 \\
520\end{array}$ & $\begin{array}{l}32.3 \\
38.9 \\
37.7\end{array}$ & $\begin{array}{r}94 \\
117 \\
125\end{array}$ \\
\hline J. A. W.* & $\begin{array}{l}\text { Room air } \\
3 \% \mathrm{CO}_{2} \\
5 \% \mathrm{CO}_{2}\end{array}$ & $\begin{array}{l}-77 \\
30\end{array}$ & $\begin{array}{l}9 \\
12 \\
13.7\end{array}$ & $\begin{array}{l}0.75 \\
0.76 \\
0.76\end{array}$ & $\begin{array}{l}127 \\
117 \\
149\end{array}$ & $\begin{array}{r}595 \\
838 \\
1,420\end{array}$ & $\begin{array}{r}5.36 \\
10.07 \\
19.48\end{array}$ & $\begin{array}{l}218 \\
311 \\
408\end{array}$ & $\begin{array}{l}36.7 \\
37.1 \\
28.7\end{array}$ & $\begin{array}{r}96 \\
116 \\
126\end{array}$ \\
\hline J. K. A. & $\begin{array}{l}\text { Room air } \\
3 \% \mathrm{CO}_{2} \\
5 \% \mathrm{CO}_{2}\end{array}$ & $\begin{array}{l}\overline{26} \\
32\end{array}$ & $\begin{array}{l}11 \\
11 \\
13.7\end{array}$ & $\begin{array}{l}0.73 \\
0.73 \\
0.77\end{array}$ & $\begin{array}{r}96 \\
122 \\
149\end{array}$ & $\begin{array}{r}433 \\
836 \\
1,274\end{array}$ & $\begin{array}{r}4.76 \\
9.20 \\
17.43\end{array}$ & $\begin{array}{l}149 \\
170 \\
161\end{array}$ & $\begin{array}{l}34.3 \\
20.3 \\
12.6\end{array}$ & $\begin{array}{r}96 \\
115 \\
124\end{array}$ \\
\hline H. J.* & $\begin{array}{l}\text { Room air } \\
3 \% \mathrm{CO}_{2} \\
5 \% \mathrm{CO}_{2}\end{array}$ & $\begin{array}{l}- \\
29 \\
29\end{array}$ & $\begin{array}{l}18 \\
21.3 \\
22.3\end{array}$ & $\begin{array}{l}0.86 \\
0.83 \\
0.76\end{array}$ & $\begin{array}{l}137 \\
139 \\
125\end{array}$ & $\begin{array}{l}425 \\
600 \\
912\end{array}$ & $\begin{array}{r}7.64 \\
12.80 \\
20.35\end{array}$ & $\begin{array}{l}170 \\
175 \\
380\end{array}$ & $\begin{array}{l}39.9 \\
29.2 \\
41.7\end{array}$ & $\begin{array}{l}100 \\
117 \\
124\end{array}$ \\
\hline J. D. & $\begin{array}{l}\text { Room air } \\
3 \% \mathrm{CO}_{2} \\
5 \% \mathrm{CO}_{2}\end{array}$ & $\begin{array}{l}- \\
28 \\
28\end{array}$ & $\begin{array}{l}15.3 \\
17 \\
19\end{array}$ & $\begin{array}{l}0.74 \\
0.74 \\
0.84\end{array}$ & $\begin{array}{l}140 \\
118 \\
125\end{array}$ & $\begin{array}{l}422 \\
586 \\
729\end{array}$ & $\begin{array}{r}6.44 \\
9.96 \\
13.87\end{array}$ & $\begin{array}{l}185 \\
237 \\
148\end{array}$ & $\begin{array}{l}43.8 \\
40.6 \\
20.3\end{array}$ & $\begin{array}{r}91 \\
111 \\
122\end{array}$ \\
\hline H. M. & $\begin{array}{l}\text { Room air } \\
3 \% \mathrm{CO}_{2} \\
5 \% \mathrm{CO}_{2}\end{array}$ & $\begin{array}{l}-26 \\
28\end{array}$ & $\begin{array}{l}24.3 \\
25 \\
27.7\end{array}$ & $\begin{array}{l}0.83 \\
0.96 \\
0.84\end{array}$ & $\begin{array}{l}120 \\
108 \\
115\end{array}$ & $\begin{array}{l}296 \\
504 \\
658\end{array}$ & $\begin{array}{r}7.09 \\
12.60 \\
18.24\end{array}$ & $\begin{array}{l}149 \\
217 \\
300\end{array}$ & $\begin{array}{l}50.6 \\
43.1 \\
45.6\end{array}$ & $\begin{array}{r}92 \\
115 \\
120\end{array}$ \\
\hline P. P. & $\begin{array}{l}\text { Room air } \\
3 \% \mathrm{CO}_{2} \\
5 \% \mathrm{CO}_{2}\end{array}$ & $\begin{array}{l}-26 \\
26\end{array}$ & $\begin{array}{l}19.7 \\
25.7 \\
26.3\end{array}$ & $\begin{array}{l}0.83 \\
0.95 \\
0.74\end{array}$ & $\begin{array}{l}107 \\
112 \\
123\end{array}$ & $\begin{array}{l}282 \\
471 \\
593\end{array}$ & $\begin{array}{r}5.53 \\
12.10 \\
15.60\end{array}$ & $\begin{array}{l}129 \\
195 \\
227\end{array}$ & $\begin{array}{l}45.8 \\
41.5 \\
38.4\end{array}$ & $\begin{array}{r}91 \\
118 \\
122\end{array}$ \\
\hline V. M. & $\begin{array}{l}\text { Room air } \\
3 \% \mathrm{CO}_{2} \\
5 \% \mathrm{CO}_{2}\end{array}$ & $\begin{array}{l}-25 \\
27\end{array}$ & $\begin{array}{r}9.3 \\
13.7 \\
17.7\end{array}$ & $\begin{array}{l}0.77 \\
0.86 \\
0.77\end{array}$ & $\begin{array}{r}96 \\
141 \\
121\end{array}$ & $\begin{array}{r}424 \\
959 \\
1,193\end{array}$ & $\begin{array}{r}3.94 \\
13.14 \\
21.13\end{array}$ & $\begin{array}{l}133 \\
246 \\
382\end{array}$ & $\begin{array}{l}31.3 \\
25.7 \\
32.1\end{array}$ & $\begin{array}{r}97 \\
120 \\
127\end{array}$ \\
\hline N. B. & $\begin{array}{l}\text { Room air } \\
3 \% \mathrm{CO}_{2} \\
5 \% \mathrm{CO}_{2}\end{array}$ & $\begin{array}{l}- \\
30 \\
26\end{array}$ & $\begin{array}{l}15.3 \\
24 \\
21.3\end{array}$ & $\begin{array}{l}0.86 \\
0.96 \\
0.85\end{array}$ & $\begin{array}{l}110 \\
105 \\
116\end{array}$ & $\begin{array}{l}419 \\
549 \\
922\end{array}$ & $\begin{array}{r}6.42 \\
13.17 \\
19.68\end{array}$ & $\begin{array}{l}164 \\
171 \\
201\end{array}$ & $\begin{array}{l}39.2 \\
31.2 \\
21.8\end{array}$ & $\begin{array}{l}105 \\
124 \\
128\end{array}$ \\
\hline B. 0 . & $\begin{array}{l}\text { Room air } \\
3 \% \mathrm{CO}_{2} \\
5 \% \mathrm{CO}_{2}\end{array}$ & $\begin{array}{l}-\overline{30} \\
31\end{array}$ & $\begin{array}{l}15 \\
17.3 \\
18.3\end{array}$ & $\begin{array}{l}0.83 \\
0.88 \\
0.77\end{array}$ & $\begin{array}{l}115 \\
118 \\
114\end{array}$ & $\begin{array}{l}405 \\
626 \\
948\end{array}$ & $\begin{array}{r}6.07 \\
10.83 \\
17.35\end{array}$ & $\begin{array}{l}166 \\
162 \\
116\end{array}$ & $\begin{array}{l}41 \\
26 \\
12\end{array}$ & $\begin{array}{l}101 \\
120 \\
128\end{array}$ \\
\hline M. B. & $\begin{array}{l}\text { Room air } \\
3 \% \mathrm{CO}_{2} \\
5 \% \mathrm{CO}_{2}\end{array}$ & $\begin{array}{l}\overline{33} \\
37\end{array}$ & $\begin{array}{l}20.7 \\
23 \\
22.3\end{array}$ & $\begin{array}{l}0.77 \\
0.80 \\
0.70\end{array}$ & $\begin{array}{l}116 \\
109 \\
111\end{array}$ & $\begin{array}{l}272 \\
376 \\
632\end{array}$ & $\begin{array}{r}5.62 \\
8.64 \\
14.10\end{array}$ & $\begin{array}{l}128 \\
136 \\
181\end{array}$ & $\begin{array}{l}47.2 \\
36.1 \\
28.7\end{array}$ & $\begin{array}{r}99 \\
117 \\
126\end{array}$ \\
\hline
\end{tabular}

* Large dead space flutter valve used.

changes in ventilation may be'related to these concentrations after the manner of Gray (17). Average values for arterial blood $\mathrm{pH}$ were 7.42 at rest, 7.41 with 3 per cent $\mathrm{CO}_{2}$ inhalation, and 7.38 with 5 per cent $\mathrm{CO}_{2}$. Hydrogen ion concentrations expressed as billionths of moles per liter corresponding to the above $\mathrm{pH}$ values are $37.9,38.8$, and 41.7, respectively. Average values for arterial $\mathrm{CO}_{2}$ tension in $\mathrm{mm}$. $\mathrm{Hg}$ were 41.5 at rest, 44 with 3 per cent $\mathrm{CO}_{2}$ inhalation, and 46.5 with 5 per cent $\mathrm{CO}_{2}$. Although tending to rise with
$\mathrm{CO}_{2}$ inhalation, the changes in oxygen saturation of the arterial blood were somewhat less consistent, presumably because of inability to measure accurately with the Van Slyke technique the small changes in oxygen saturation associated with increased oxygen tension in this region of the dissociation curve. Arterial hematocrit showed no appreciable change. No direct observations on urinary constituents were made to evaluate possible renal adjustments to the respiratory acidosis induced during the thirty-minute period of $\mathrm{CO}_{2}$ 
breathing. However, the absence of any appreciable change in the whole blood buffer base at the end of the $\mathrm{CO}_{2}$ inhalation period suggests that such adjustments as may have occurred were not significant.

Observations on changes in effective alveolar ventilation, and correlation of these changes with hydrogen ion concentration and $\mathrm{CO}_{2}$ tension in the arterial blood are described in Table V. If the effective alveolar ventilation is measured in terms of liters per minute per square meter body surface area, the average value at rest is 1.93 .
Body surface area has been arbitrarily chosen here as a reference point which would permit some comparison of alveolar ventilation, oxygen consumption, and sensitivity to chemical respiratory stimuli among the normal subjects and the other groups studied. The age range in this group of normal subjects was 17 to 43 years, average age 30.4. Robinson's data (18) for the closest average age group (35.1 years) yield a figure of 2.07 liters per minute per square meter body surface area for effective alveolar ventilation. Typical stimulus-response curves or sensitivity slopes of a

TABLE IV

Physiologic data relative to the arterial blood at rest and during $\mathrm{CO}_{2}$ inhalation in twelve normal subjects

\begin{tabular}{|c|c|c|c|c|c|c|c|c|}
\hline Subject & $\begin{array}{l}\text { Inspired } \\
\text { gas } \\
\text { mixture }\end{array}$ & pHs & $\left(H^{+}\right)_{2}$ & $\mathrm{Cs}_{\mathrm{CO}_{2}}$ & $\mathrm{~Pa}_{\mathrm{OO}_{2}}$ & $\mathrm{Sa}_{\mathrm{O}}$ & Vo & $\underset{\left(\mathbf{B}_{\mathbf{B}}+\right)_{\mathrm{b}}}{\text { Calc. }}$ \\
\hline T.J. & $\begin{array}{l}\text { Room air } \\
3 \% \mathrm{CO}_{2} \\
5 \% \mathrm{CO}_{2}\end{array}$ & $\begin{array}{l}7.42 \\
7.41 \\
7.37\end{array}$ & $\begin{array}{l}38.0 \\
38.9 \\
42.6\end{array}$ & $\begin{array}{l}\mathbf{5 8 . 1} \\
\mathbf{5 8 . 6} \\
\mathbf{5 9 . 2}\end{array}$ & $\begin{array}{l}39 \\
40.5 \\
45\end{array}$ & $\begin{array}{l}92 \\
97 \\
95\end{array}$ & $\begin{array}{l}33 \\
33 \\
34\end{array}$ & $\begin{array}{l}47 \\
47 \\
47\end{array}$ \\
\hline J. R. W. & $\begin{array}{l}\text { Room air } \\
3 \% \mathrm{CO}_{2} \\
5 \% \mathrm{CO}_{2}\end{array}$ & $\begin{array}{l}7.42 \\
7.41 \\
7.39\end{array}$ & $\begin{array}{l}38.0 \\
38.9 \\
40.7\end{array}$ & $\begin{array}{l}66.3 \\
67.0 \\
67.8\end{array}$ & $\begin{array}{l}45 \\
46.5 \\
49.5\end{array}$ & $\begin{array}{r}96 \\
96 \\
100\end{array}$ & $\begin{array}{l}41 \\
42 \\
41\end{array}$ & $\begin{array}{l}51 \\
51 \\
51\end{array}$ \\
\hline J.A. W. & $\begin{array}{l}\text { Room air } \\
3 \% \mathrm{CO}_{2} \\
5 \% \mathrm{CO}_{2}\end{array}$ & $\begin{array}{l}7.40 \\
7.39 \\
7.37\end{array}$ & $\begin{array}{l}39.8 \\
40.7 \\
42.7\end{array}$ & $\begin{array}{l}61.4 \\
61.8 \\
63.5\end{array}$ & $\begin{array}{l}43 \\
45 \\
48\end{array}$ & $\begin{array}{l}98 \\
98 \\
99\end{array}$ & $\begin{array}{l}42 \\
42 \\
43\end{array}$ & $\begin{array}{l}49 \\
49 \\
49\end{array}$ \\
\hline J. K. A. & $\begin{array}{l}\text { Room air } \\
3 \% \mathrm{CO}_{2} \\
5 \% \mathrm{CO}_{2}\end{array}$ & $\begin{array}{l}7.45 \\
7.42 \\
7.40\end{array}$ & $\begin{array}{l}35.5 \\
38.0 \\
39.8\end{array}$ & $\begin{array}{l}64.1 \\
64.6 \\
66.3\end{array}$ & $\begin{array}{l}40.5 \\
43 \\
47.5\end{array}$ & $\begin{array}{r}97 \\
100 \\
100\end{array}$ & $\begin{array}{l}37 \\
37 \\
39\end{array}$ & $\begin{array}{l}50 \\
50 \\
50\end{array}$ \\
\hline H. J. & $\begin{array}{l}\text { Room air } \\
3 \% \mathrm{CO}_{2} \\
5 \% \mathrm{CO}_{2}\end{array}$ & $\begin{array}{l}7.42 \\
7.41 \\
7.40\end{array}$ & $\begin{array}{l}38.0 \\
38.9 \\
39.8\end{array}$ & $\begin{array}{l}63.3 \\
63.5 \\
64.1\end{array}$ & $\begin{array}{l}42.5 \\
43.5 \\
45\end{array}$ & $\begin{array}{l}99 \\
98 \\
97\end{array}$ & $\begin{array}{l}40 \\
40 \\
41\end{array}$ & $\begin{array}{l}50 \\
50 \\
50\end{array}$ \\
\hline J. D. & $\begin{array}{l}\text { Room air } \\
3 \% \mathrm{CO}_{2} \\
5 \% \mathrm{CO}_{2}\end{array}$ & $\begin{array}{l}7.44 \\
7.42 \\
7.40\end{array}$ & $\begin{array}{l}36.3 \\
38.0 \\
39.8\end{array}$ & $\begin{array}{l}67.3 \\
66.1 \\
66.4\end{array}$ & $\begin{array}{l}43 \\
44.5 \\
47\end{array}$ & $\begin{array}{l}97 \\
99 \\
96\end{array}$ & $\begin{array}{l}41 \\
41 \\
42\end{array}$ & $\begin{array}{l}52 \\
51 \\
51\end{array}$ \\
\hline H. M. & $\begin{array}{l}\text { Room air } \\
3 \% \mathrm{CO}_{2} \\
5 \% \mathrm{CO}_{2}\end{array}$ & $\begin{array}{l}7.40 \\
7.39 \\
7.37\end{array}$ & $\begin{array}{l}39.8 \\
40.7 \\
42.7\end{array}$ & $\begin{array}{l}62.7 \\
62.7 \\
63.6\end{array}$ & $\begin{array}{l}44.5 \\
46 \\
48\end{array}$ & $\begin{array}{l}96 \\
96 \\
96\end{array}$ & $\begin{array}{l}41 \\
42 \\
42\end{array}$ & $\begin{array}{l}49 \\
50 \\
49\end{array}$ \\
\hline P. P. & $\begin{array}{l}\text { Room air } \\
3 \% \mathrm{CO}_{2} \\
5 \% \mathrm{CO}_{2}\end{array}$ & $\begin{array}{l}7.42 \\
7.41 \\
7.39\end{array}$ & $\begin{array}{l}38.0 \\
38.9 \\
40.7\end{array}$ & $\begin{array}{l}64.9 \\
66.4 \\
64.7\end{array}$ & $\begin{array}{l}43.5 \\
45.5 \\
47\end{array}$ & $\begin{array}{l}95 \\
95 \\
97\end{array}$ & $\begin{array}{l}39 \\
39 \\
40\end{array}$ & $\begin{array}{l}50 \\
51 \\
49\end{array}$ \\
\hline V. M. & $\begin{array}{l}\text { Room air } \\
3 \% \mathrm{CO}_{2} \\
5 \% \mathrm{CO}_{2}\end{array}$ & $\begin{array}{l}7.40 \\
7.39 \\
7.38\end{array}$ & $\begin{array}{l}39.8 \\
40.7 \\
41.7\end{array}$ & $\begin{array}{l}56.1 \\
57.7 \\
57.1\end{array}$ & $\begin{array}{l}39.5 \\
41.5 \\
42.5\end{array}$ & $\begin{array}{l}97 \\
98 \\
99\end{array}$ & $\begin{array}{l}42 \\
43 \\
43\end{array}$ & $\begin{array}{l}47 \\
47 \\
46\end{array}$ \\
\hline N. B. & $\begin{array}{l}\text { Room air } \\
3 \% \mathrm{CO}_{2} \\
5 \% \mathrm{CO}_{2}\end{array}$ & $\begin{array}{l}7.43 \\
7.40 \\
7.36\end{array}$ & $\begin{array}{l}37.2 \\
39.8 \\
43.7\end{array}$ & $\begin{array}{l}\mathbf{5 5 . 4} \\
56.6 \\
59.6\end{array}$ & $\begin{array}{l}37 \\
40 \\
46\end{array}$ & $\begin{array}{l}98 \\
99 \\
99\end{array}$ & $\begin{array}{l}37 \\
38 \\
38\end{array}$ & $\begin{array}{l}47 \\
47 \\
47\end{array}$ \\
\hline B. 0 . & $\begin{array}{l}\text { Room air } \\
3 \% \mathrm{CO}_{2} \\
5 \% \mathrm{CO}_{2}\end{array}$ & $\begin{array}{l}7.44 \\
7.42 \\
7.40\end{array}$ & $\begin{array}{l}36.3 \\
38.0 \\
39.8\end{array}$ & $\begin{array}{l}60.3 \\
65.1 \\
64.9\end{array}$ & $\begin{array}{l}40.5 \\
43.5 \\
45.5\end{array}$ & $\begin{array}{l}98 \\
96 \\
97\end{array}$ & $\begin{array}{l}47 \\
47 \\
48\end{array}$ & $\begin{array}{l}\mathbf{5 1} \\
\mathbf{5 1} \\
\mathbf{5 1}\end{array}$ \\
\hline M. B. & $\begin{array}{l}\text { Room air } \\
3 \% \mathrm{CO}_{2} \\
5 \% \mathrm{CO}_{2}\end{array}$ & $\begin{array}{l}7.43 \\
7.39 \\
7.35\end{array}$ & $\begin{array}{l}37.2 \\
40.7 \\
44.7\end{array}$ & $\begin{array}{l}59.6 \\
61.6 \\
60.6\end{array}$ & $\begin{array}{l}40 \\
44.5 \\
47\end{array}$ & $\begin{array}{l}99 \\
99 \\
96\end{array}$ & $\begin{array}{l}37 \\
36 \\
36\end{array}$ & $\begin{array}{l}49 \\
48 \\
47\end{array}$ \\
\hline
\end{tabular}


TABLE v

Physiologic data relative to effective alveolar ventilation and sensitivity to the carbon dioxide-hydrogen ion stimulus in twelve normal subjects

\begin{tabular}{|c|c|c|c|c|c|c|c|c|}
\hline Subject & $\begin{array}{c}\text { Inspired } \\
\text { gas } \\
\text { mixture }\end{array}$ & $\dot{\mathbf{v}}_{\mathbf{A}}$ & $\dot{V}_{\mathbf{A} / \mathbf{M}^{2}}$ & VR & $\frac{\mathrm{Pa}_{\mathrm{OO}_{2}}}{\mathrm{VR}}$ & $\frac{\dot{\mathrm{V}}_{\mathbf{A} / \mathrm{M}_{2}}}{\mathrm{~Pa}_{\mathrm{CO}_{2}}}$ & $\frac{\left(H^{+}\right) a}{V R}$ & $\frac{\dot{\mathrm{V}}_{\Lambda} / \mathrm{M}^{2}}{\left(\mathrm{H}^{+}\right) \mathrm{a}}$ \\
\hline T. J. & $\begin{array}{l}\text { Room air } \\
3 \% \mathrm{CO}_{2} \\
5 \% \mathrm{CO}_{2}\end{array}$ & $\begin{array}{l}3.86 \\
9.40 \\
19 .\end{array}$ & $\begin{array}{l}1.94 \\
4.72 \\
9.55\end{array}$ & $\begin{array}{l}1 \\
2.33 \\
4.92\end{array}$ & 1.5 & 1.4 & 1.2 & 1.8 \\
\hline J. R. W. & $\begin{array}{l}\text { Room air } \\
3 \% \mathrm{CO}_{2} \\
5 \% \mathrm{CO}_{2}\end{array}$ & $\begin{array}{l}3.48 \\
7.43 \\
12 .\end{array}$ & $\begin{array}{l}1.82 \\
3.89 \\
6.28\end{array}$ & $\begin{array}{l}1 \\
2.13 \\
3.44\end{array}$ & 1.7 & 1.2 & 1.1 & 1.9 \\
\hline J.A. W. & $\begin{array}{l}\text { Room air } \\
3 \% \mathrm{CO}_{2} \\
5 \% \mathrm{CO}_{2}\end{array}$ & $\begin{array}{r}3.38 \\
6.33 \\
13.88\end{array}$ & $\begin{array}{l}1.72 \\
3.23 \\
7.08\end{array}$ & $\begin{array}{l}1 \\
1.87 \\
4.12\end{array}$ & 1.6 & 1.1 & 0.9 & 1.9 \\
\hline J. K. A. & $\begin{array}{l}\text { Room air } \\
3 \% \mathrm{CO}_{2} \\
5 \% \mathrm{CO}_{2}\end{array}$ & $\begin{array}{r}3.13 \\
7.33 \\
15.25\end{array}$ & $\begin{array}{l}1.49 \\
3.49 \\
7.26\end{array}$ & $\begin{array}{l}1 \\
2.33 \\
4.87\end{array}$ & 1.8 & 0.9 & 1.2 & 1.5 \\
\hline H. J. & $\begin{array}{l}\text { Room air } \\
3 \% \mathrm{CO}_{2} \\
5 \% \mathrm{CO}_{2}\end{array}$ & $\begin{array}{r}4.58 \\
9.05 \\
11.83\end{array}$ & $\begin{array}{l}2.42 \\
4.79 \\
6.26\end{array}$ & $\begin{array}{l}1 \\
1.97 \\
2.58\end{array}$ & 1.5 & 1.6 & 1.1 & 2.2 \\
\hline J. D. & $\begin{array}{l}\text { Room air } \\
3 \% \mathrm{CO}_{2} \\
5 \% \mathrm{CO}_{2}\end{array}$ & $\begin{array}{r}3.62 \\
5.91 \\
11.10\end{array}$ & $\begin{array}{l}2.09 \\
3.42 \\
6.42\end{array}$ & $\begin{array}{l}1 \\
1.63 \\
3.06\end{array}$ & 2.0 & 1.0 & 1.7 & 1.1 \\
\hline H. M. & $\begin{array}{l}\text { Room air } \\
3 \% \mathrm{CO}_{2} \\
5 \% \mathrm{CO}_{2}\end{array}$ & $\begin{array}{l}3.50 \\
7.17 \\
9.92\end{array}$ & $\begin{array}{l}1.96 \\
4.01 \\
5.54\end{array}$ & $\begin{array}{l}1 \\
2.05 \\
2.84\end{array}$ & 1.9 & 1.1 & 1.5 & 1.4 \\
\hline P. P. & $\begin{array}{l}\text { Room air } \\
3 \% \mathrm{CO}_{2} \\
5 \% \mathrm{CO}_{2}\end{array}$ & $\begin{array}{l}3 . \\
7.07 \\
9.60\end{array}$ & $\begin{array}{l}1.79 \\
4.21 \\
5.71\end{array}$ & $\begin{array}{l}1 \\
2.36 \\
3.19\end{array}$ & 1.5 & 1.2 & 1.1 & 1.7 \\
\hline V. M. & $\begin{array}{l}\text { Room air } \\
3 \% \mathrm{CO}_{2} \\
5 \% \mathrm{CO}_{2}\end{array}$ & $\begin{array}{r}2.71 \\
9.75 \\
14.30\end{array}$ & $\begin{array}{l}1.62 \\
5.84 \\
8.56\end{array}$ & $\begin{array}{l}1 \\
3.60 \\
5.27\end{array}$ & 0.8 & 2.3 & 0.5 & 3.8 \\
\hline N. B. & $\begin{array}{l}\text { Room air } \\
3 \% \mathrm{CO}_{2} \\
5 \% \mathrm{CO}_{2}\end{array}$ & $\begin{array}{r}3.90 \\
9.06 \\
15.40\end{array}$ & $\begin{array}{l}2.30 \\
5.33 \\
9.06\end{array}$ & $\begin{array}{l}1 \\
2.32 \\
3.95\end{array}$ & 2.7 & 0.9 & 2.0 & 1.1 \\
\hline B. 0 . & $\begin{array}{l}\text { Room air } \\
3 \% \mathrm{CO}_{2} \\
5 \% \mathrm{CO}_{2}\end{array}$ & $\begin{array}{r}3.58 \\
8.02 \\
15.23\end{array}$ & $\begin{array}{l}2.03 \\
4.56 \\
8.65\end{array}$ & $\begin{array}{l}1 \\
2.24 \\
4.25\end{array}$ & 1.5 & 1.3 & 0.9 & 2.2 \\
\hline \multirow[t]{3}{*}{ M. B. } & $\begin{array}{l}\text { Room air } \\
3 \% \mathrm{CO}_{2} \\
5 \% \mathrm{CO}_{2}\end{array}$ & $\begin{array}{r}2.97 \\
5.53 \\
10.04\end{array}$ & $\begin{array}{l}1.95 \\
3.64 \\
6.60\end{array}$ & $\begin{array}{l}1 \\
1.86 \\
3.38\end{array}$ & 2.2 & 0.8 & 2.9 & 0.7 \\
\hline & & & \multirow{2}{*}{\multicolumn{2}{|c|}{$\begin{array}{l}\text { Average: } \\
\text { Range } \\
\text { S.D. }\end{array}$}} & $\begin{array}{l}1.7 \\
0.8-2.7\end{array}$ & 1.2 & $\begin{array}{c}1.3 \\
0.5-2.9\end{array}$ & 1.8 \\
\hline & & & & & \pm 0.5 & $\begin{array}{l}0.8-2.3 \\
\pm 0.3\end{array}$ & \pm 0.6 & $\begin{array}{l}0.7-8 \\
\pm 0.7\end{array}$ \\
\hline
\end{tabular}

normal subject, constructed in the manner previously indicated, are shown in Figures 1 and 2.

To double the effective alveolar ventilation at rest an average increase of $1.7 \mathrm{~mm}$. $\mathrm{Hg}$ (range 0.8 to $2.7, \mathrm{~S} . \mathrm{D} . \pm 0.5$ ) in arterial $\mathrm{CO}_{2}$ tension was required, associated with an average increase in arterial hydrogen ion concentration of $1.3 \times 10^{-7}$ moles per liter (range 0.5 to 2.9, S.D. \pm 0.6 ).
For each $\mathrm{mm}$. $\mathrm{Hg}$ rise in $\mathrm{CO}_{2}$ tension of the arterial blood there was an average increase in effective alveolar ventilation per square meter body surface area of 1.2 liters per minute (range 0.8 to 2.3 , S.D. \pm 0.3 ). For an increase of $1.0 \times 10^{-7}$ moles per liter in hydrogen ion concentration of the arterial blood an average increase in effective alveolar ventilation per square meter body surface 


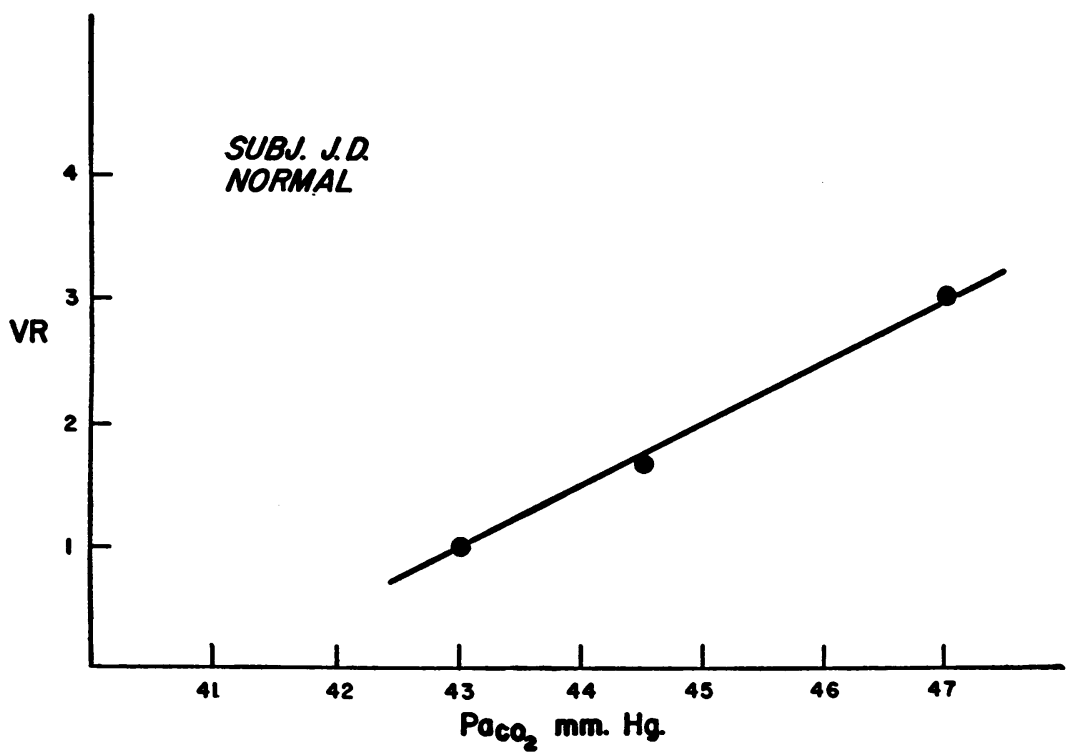

Fig. 1. Strmulus Response Curfe in a Normal Subject, Relating Arterial $\mathrm{CO}_{2}$ Tension to alveolar Ventilation Ratio

area of 1.9 liters per minute (range 0.7 to 3.8 , inhalation well, without respiratory distress. A S.D. \pm 0.7 ) occurred. few complained of mild headache following the procedure. The patients have been separated into

Pulmonary emphysema three groups according to the classification of

Thirteen patients with chronic pulmonary Baldwin, Cournand, and Richards (1). Those in emphysema were studied. All tolerated the $\mathrm{CO}_{2}$ Group II were found to have arterial oxygen un-

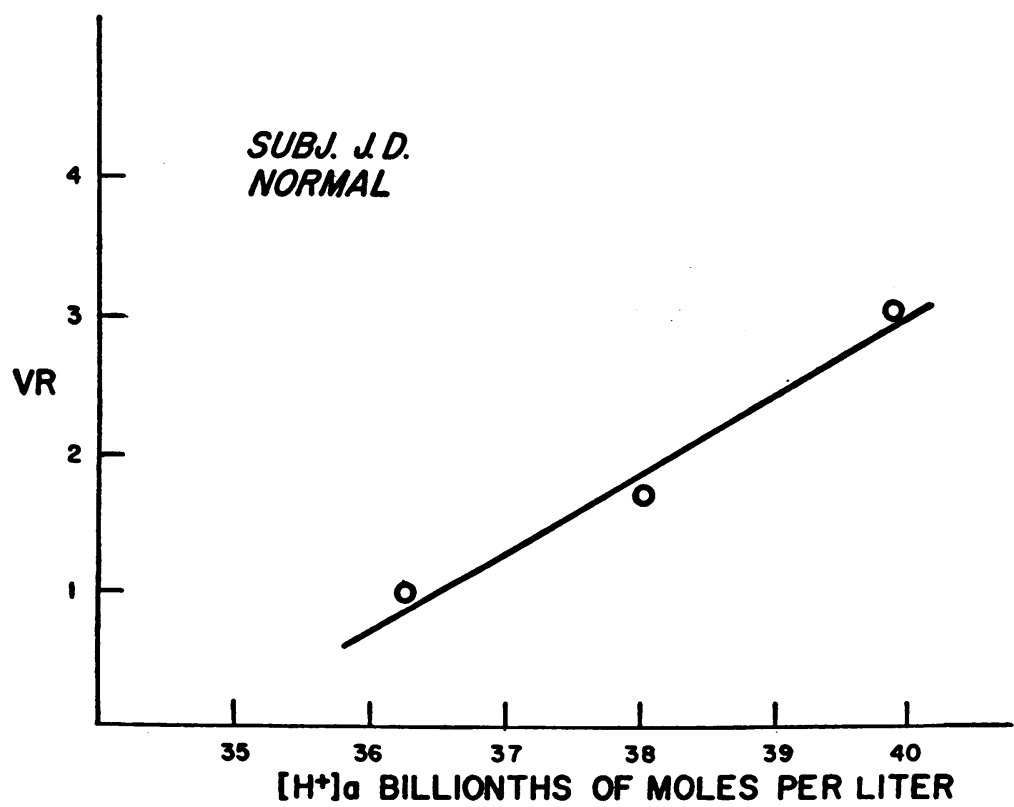

Fig. 2. Stmulus Response Curve in a Normal Subject, Relating Arterial Hydrogen Ion Concentration to Alveolar Ventilation Ratio 
TABLE VI

Physiologic data relative to pulmonary ventilation and arterial blood under conditions of rest and standard exercise in thirteen emphysematous subjects *

\begin{tabular}{|c|c|c|c|c|c|c|c|c|c|c|c|c|}
\hline \multirow{2}{*}{\multicolumn{2}{|c|}{ Subject }} & \multirow[b]{3}{*}{ B.S.A. } & \multirow{2}{*}{\multicolumn{2}{|c|}{$\dot{\mathbf{V}}_{\mathbf{X}} / \mathbf{M}^{\mathbf{2}}$}} & \multirow{2}{*}{\multicolumn{2}{|c|}{$\dot{\mathrm{V}}_{2} / \mathbf{M}^{2}$}} & \multicolumn{6}{|c|}{ Arterial blood } \\
\hline & & & & & & & \multicolumn{2}{|c|}{ pHs } & \multicolumn{2}{|c|}{$\mathrm{Sa}_{\mathrm{O}_{2}}$} & \multicolumn{2}{|c|}{$\mathrm{Pa}_{\mathrm{CO}_{2}} \mathrm{~mm} . \mathrm{Hg}$} \\
\hline Sex & Age & & Rest & Exer. & Rest & Exer. & Rest & Exer. & Rest & Exer. & Rest & Exer. \\
\hline \multicolumn{13}{|c|}{ Emphysema IV } \\
\hline $\mathbf{F}$ & 47 & 1.65 & 4.30 & - & 137 & - & 7.33 & - & 59 & - & 54 & - \\
\hline M & 47 & 1.75 & 3.45 & 11.15 & 134 & - & 7.38 & 7.28 & 78 & 75 & 54 & 64 \\
\hline & 58 & 1.64 & 5.00 & 12.60 & 160 & 306 & 7.46 & 7.42 & 87.6 & 54.8 & 45 & 50 \\
\hline $\mathbf{M}$ & 74 & 1.81 & 4.79 & 7.91 & 141 & 242 & 7.44 & 7.43 & 86 & 75.8 & 49.5 & 51.5 \\
\hline $\mathbf{M}$ & 52 & 1.79 & 3.74 & 5.88 & 139 & 207 & 7.38 & 7.32 & 81.6 & 53.8 & 59 & 66 \\
\hline $\mathbf{M}$ & 70 & 1.53 & 5.95 & 12.67 & 145 & 306 & 7.36 & 7.36 & 88.1 & 84.7 & 55 & 52 \\
\hline \multicolumn{13}{|c|}{ Emphysema III } \\
\hline M & 56 & 1.82 & 5.05 & 16.9 & 141 & 575 & 7.40 & 7.39 & 97.6 & 93 & 48 & 49 \\
\hline $\mathbf{M}$ & 75 & 1.54 & 3.99 & 10.15 & 119 & 301 & 7.39 & 7.36 & 90.4 & 86.4 & 48 & 53 \\
\hline $\mathbf{M}$ & 46 & 1.83 & 4.83 & 12.60 & 143 & 405 & 7.40 & 7.29 & 80.6 & 67.7 & 47 & 60 \\
\hline \multicolumn{13}{|c|}{ Emphysema II } \\
\hline $\mathbf{M}$ & 48 & 1.61 & 5.70 & 12.61 & 127 & 374 & 7.43 & 7.42 & 93 & 90 & 40 & 40 \\
\hline $\mathbf{M}$ & 69 & 1.46 & 7.36 & 19.60 & 166 & 486 & 7.40 & 7.40 & 89 & 89 & 41 & 37 \\
\hline $\mathbf{F}$ & 54 & 1.60 & 4.69 & 15.12 & 134 & 436 & 7.40 & 7.38 & 96.2 & 90.5 & 36 & 36.5 \\
\hline $\mathbf{M}$ & 48 & 1.89 & 5.63 & 14.47 & 133 & 402 & 7.44 & 7.45 & 92.2 & 92.7 & 36 & 35 \\
\hline
\end{tabular}

* In some instances these studies were done on a different day than those recorded in Tables VIII and IX, so that resting values are not exactly the same.

saturation at rest or following standard exercise, but no $\mathrm{CO}_{2}$ retention. In addition to anoxemia, those in Group III had elevation of the arterial $\mathrm{CO}_{2}$ tension at rest or following exercise, but no evidence of congestive heart failure. Finally, patients in Group IV suffered from anoxemia, $\mathrm{CO}_{2}$ retention and cor pulmonale, with chronic congestive failure. The data obtained relative to ventilation, oxygen consumption, and arterial blood in these patients under conditions of rest and exercise appear in Table VI. Indices of intrapulmonary mixing, lung volumes, maximum breathing capacities, and serum electrolytes where available, are shown in Table VII. All were found to have reductions in vital and maximum breathing capacities. Both the index of intrapulmonary gas mixing and the residual volume to total capacity ratio were increased in all instances except one.
The response of the emphysematous subjects to the $\mathrm{CO}_{2}$ stimulus was determined in the same manner as was done with the normal individuals. The data so derived are shown in Tables VIII, IX, and X. Similar results were obtained in Groups III and IV, and they may be considered together. Certain of these results, with the average normal values for comparison, are summarized in Table XI. As indicated by the respiratory exchange ratios in Table VIII, it was possible to achieve steady states during $\mathrm{CO}_{2}$ inhalation which were comparable to those in normal individuals. The average percentage increases in tidal volume and total pulmonary ventilation were approximately half the normal values. As in the normal group, there was little change in oxygen consumption with $\mathrm{CO}_{2}$ inhalation, but the average oxygen consumption of $137 \mathrm{ml}$. per square meter body surface area at rest for the emphysematous pa- 

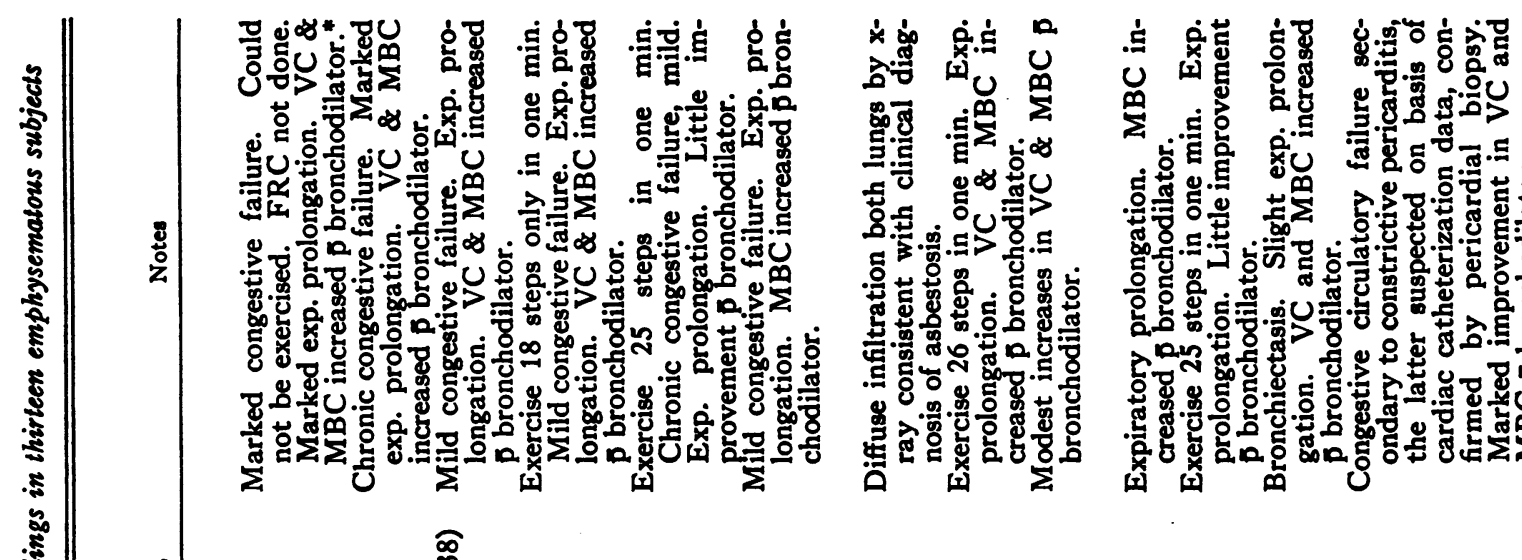

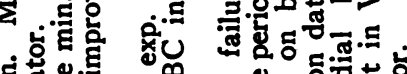

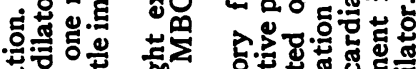

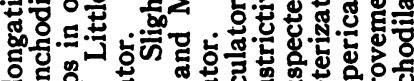

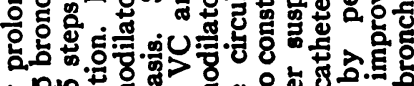

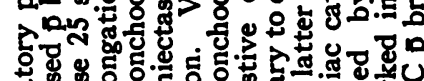

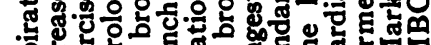

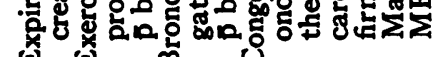

紊 $\pi$ 的

登宫 ( 1 i 111

$\stackrel{?}{\mathbb{Z}}$

紊

焉

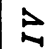

है

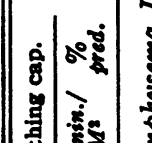
路 竞 $\stackrel{\infty}{=}$ ลे $\exists$

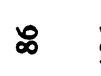

8 क

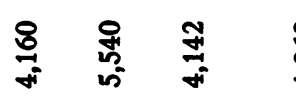

8

๘

$\approx \cong \Xi$

$\Xi$

is 8

8

点 兽 胥

$\stackrel{\infty}{\infty}$

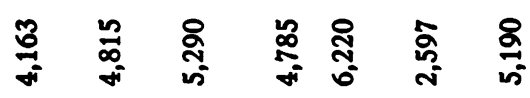

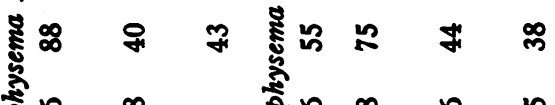

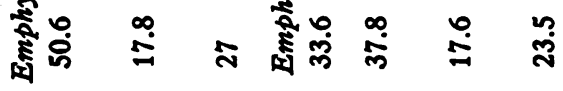

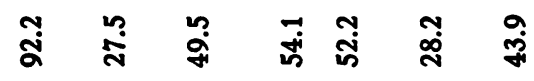

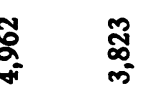
๑ ถิ $\rightarrow \sqrt{4}$

$\underset{\Sigma}{\dot{4}}$

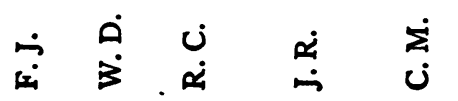

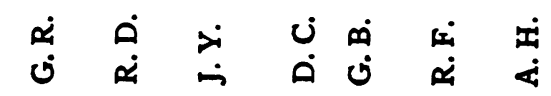


TABLE VIII

Physiologic data relative to pulmonary ventilation at rest and during $\mathrm{CO}_{2}$ inhalation in thirteen emphysematous subjects

\begin{tabular}{|c|c|c|c|c|c|c|c|c|c|c|}
\hline \multirow[b]{2}{*}{ Subject } & \multirow{2}{*}{$\begin{array}{l}\text { Inspired } \\
\text { gas } \\
\text { mixture }\end{array}$} & \multirow{2}{*}{$\begin{array}{l}\text { Time on } \\
\text { mixture } \\
\text { (min.) }\end{array}$} & \multirow[b]{2}{*}{ f } & \multirow[b]{2}{*}{$\mathbf{R} \mathbf{R}$} & \multirow[b]{2}{*}{$\dot{V}_{0} / M^{2}$} & \multirow[b]{2}{*}{$\mathbf{V}_{\mathbf{T}}$} & \multirow[b]{2}{*}{$\dot{\mathbf{V}}_{\mathbf{E}}$} & \multicolumn{2}{|c|}{ VD } & \multirow{2}{*}{$\begin{array}{l}\text { Calc. } \\
\text { PAf }^{2}\end{array}$} \\
\hline & & & & & & & & $m l$. & $\% \dot{V}_{\mathbf{E}}$ & \\
\hline \multicolumn{11}{|c|}{ Emphysema IV } \\
\hline A. M. & $\begin{array}{l}\text { Room air } \\
3 \% \mathrm{CO}_{2} \\
5 \% \mathrm{CO}_{2}\end{array}$ & $\begin{array}{l}30 \text { min. } \\
24 \mathrm{~min} .\end{array}$ & $\begin{array}{l}21.3 \\
24 \\
24\end{array}$ & $\begin{array}{l}0.73 \\
0.70 \\
0.67\end{array}$ & $\begin{array}{l}137 \\
145 \\
178\end{array}$ & $\begin{array}{l}332 \\
392 \\
563\end{array}$ & $\begin{array}{r}7.10 \\
9.41 \\
13.50\end{array}$ & $\begin{array}{l}207 \\
226 \\
322\end{array}$ & $\begin{array}{l}62.3 \\
57.6 \\
57.2\end{array}$ & $\begin{array}{r}80 \\
97 \\
103\end{array}$ \\
\hline F. J. & $\begin{array}{l}\text { Room air } \\
3 \% \mathrm{CO}_{2} \\
4 \% \mathrm{CO}_{2}\end{array}$ & $\begin{array}{l}28 \text { min. } \\
33 \text { min. }\end{array}$ & $\begin{array}{l}15.7 \\
16.3 \\
17.8\end{array}$ & $\begin{array}{l}0.70 \\
0.83 \\
0.65\end{array}$ & $\begin{array}{l}131 \\
118 \\
143\end{array}$ & $\begin{array}{l}366 \\
610 \\
656\end{array}$ & $\begin{array}{r}5.74 \\
9.95 \\
11.68\end{array}$ & $\begin{array}{l}163 \\
302 \\
328\end{array}$ & $\begin{array}{l}44.6 \\
49.5 \\
50.2\end{array}$ & $\begin{array}{r}88 \\
107 \\
109\end{array}$ \\
\hline W. D. & $\begin{array}{l}\text { Room air } \\
3 \% \mathrm{CO}_{2} \\
4 \% \mathrm{CO}_{2}\end{array}$ & $\begin{array}{l}26 \mathrm{~min} . \\
26 \mathrm{~min} .\end{array}$ & $\begin{array}{l}17 \\
17.2 \\
21.2\end{array}$ & $\begin{array}{l}0.76 \\
0.80 \\
0.76\end{array}$ & $\begin{array}{l}160 \\
153 \\
179\end{array}$ & $\begin{array}{l}483 \\
683 \\
691\end{array}$ & $\begin{array}{r}8.20 \\
11.74 \\
14.62\end{array}$ & $\begin{array}{l}253 \\
317 \\
306\end{array}$ & $\begin{array}{l}52.3 \\
46.3 \\
44.2\end{array}$ & $\begin{array}{r}91 \\
107 \\
111\end{array}$ \\
\hline R. C. & $\begin{array}{l}\text { Room air } \\
3 \% \mathrm{CO}_{2} \\
5 \% \mathrm{CO}_{2}\end{array}$ & $\begin{array}{l}30 \mathrm{~min} . \\
25 \mathrm{~min} .\end{array}$ & $\begin{array}{l}23.7 \\
24.7 \\
26.7\end{array}$ & $\begin{array}{l}0.73 \\
0.73 \\
0.64\end{array}$ & $\begin{array}{l}141 \\
137 \\
163\end{array}$ & $\begin{array}{l}366 \\
483 \\
572\end{array}$ & $\begin{array}{r}8.67 \\
11.92 \\
15.28\end{array}$ & $\begin{array}{l}227 \\
293 \\
349\end{array}$ & $\begin{array}{l}62.1 \\
60.6 \\
61.0\end{array}$ & $\begin{array}{r}84 \\
98 \\
100\end{array}$ \\
\hline J. R. & $\begin{array}{l}\text { Room air } \\
3 \% \mathrm{CO}_{2} \\
5 \% \mathrm{CO}_{2}\end{array}$ & $\begin{array}{l}32 \text { min. } \\
30 \text { min. }\end{array}$ & $\begin{array}{l}13.7 \\
15 \\
15.7\end{array}$ & $\begin{array}{l}0.79 \\
0.81 \\
0.84\end{array}$ & $\begin{array}{l}139 \\
130 \\
125\end{array}$ & $\begin{array}{l}483 \\
543 \\
655\end{array}$ & $\begin{array}{r}6.62 \\
8.15 \\
10.28\end{array}$ & $\begin{array}{l}275 \\
288 \\
344\end{array}$ & $\begin{array}{l}\mathbf{5 7 . 0} \\
\mathbf{5 3 . 0} \\
\mathbf{5 2 . 5}\end{array}$ & $\begin{array}{r}77 \\
93 \\
102\end{array}$ \\
\hline C. $\mathbf{M}$. & $\begin{array}{l}\text { Room air } \\
3 \% \mathrm{CO}_{2} \\
5 \% \mathrm{CO}_{2}\end{array}$ & $\begin{array}{l}25 \text { min. } \\
25 \mathrm{~min} .\end{array}$ & $\begin{array}{l}19.7 \\
25 \\
24\end{array}$ & $\begin{array}{l}0.74 \\
0.77 \\
0.78\end{array}$ & $\begin{array}{l}145 \\
128 \\
135\end{array}$ & $\begin{array}{l}462 \\
487 \\
644\end{array}$ & $\begin{array}{r}9.10 \\
12.17 \\
15.45\end{array}$ & $\begin{array}{l}329 \\
353 \\
455\end{array}$ & $\begin{array}{l}71.4 \\
72.7 \\
70.6\end{array}$ & $\begin{array}{r}79 \\
95 \\
103\end{array}$ \\
\hline \multicolumn{11}{|c|}{ Emphysema III } \\
\hline G. R. & $\begin{array}{l}\text { Room air } \\
3 \% \mathrm{CO}_{2} \\
5 \% \mathrm{CO}_{2}\end{array}$ & $\begin{array}{l}25 \text { min. } \\
22 \text { min. }\end{array}$ & $\begin{array}{l}20.67 \\
20.34 \\
21\end{array}$ & $\begin{array}{l}0.77 \\
0.79 \\
0.73\end{array}$ & $\begin{array}{l}135 \\
127 \\
153\end{array}$ & $\begin{array}{l}430 \\
653 \\
950\end{array}$ & $\begin{array}{r}8.90 \\
13.25 \\
19.95\end{array}$ & $\begin{array}{l}262 \\
387 \\
493\end{array}$ & $\begin{array}{l}60.7 \\
59.3 \\
51.8\end{array}$ & $\begin{array}{r}92 \\
111 \\
118\end{array}$ \\
\hline R. D. & $\begin{array}{l}\text { Room air } \\
3 \% \mathrm{CO}_{2} \\
5 \% \mathrm{CO}_{2}\end{array}$ & $\begin{array}{l}30 \text { min. } \\
28 \mathrm{~min} .\end{array}$ & $\begin{array}{l}13.7 \\
16.7 \\
20\end{array}$ & $\begin{array}{l}0.82 \\
0.88 \\
0.70\end{array}$ & $\begin{array}{l}119 \\
113 \\
123\end{array}$ & $\begin{array}{l}448 \\
633 \\
758\end{array}$ & $\begin{array}{r}6.14 \\
10.55 \\
15.17\end{array}$ & $\begin{array}{l}250 \\
354 \\
463\end{array}$ & $\begin{array}{l}\mathbf{5 5 . 8} \\
\mathbf{5 5 . 9} \\
\mathbf{6 1 . 2}\end{array}$ & $\begin{array}{r}91 \\
109 \\
113\end{array}$ \\
\hline J. Y. & $\begin{array}{l}\text { Room air } \\
3 \% \mathrm{CO}_{2} \\
5 \% \mathrm{CO}_{2}\end{array}$ & $\begin{array}{l}26 \text { min. } \\
25 \text { min. }\end{array}$ & $\begin{array}{l}20.3 \\
18.7 \\
20.7\end{array}$ & $\begin{array}{l}0.85 \\
0.77 \\
0.78\end{array}$ & $\begin{array}{l}128 \\
140 \\
159\end{array}$ & $\begin{array}{l}458 \\
643 \\
814\end{array}$ & $\begin{array}{l}9.30 \\
12 . \\
16.85\end{array}$ & $\begin{array}{l}286 \\
347 \\
424\end{array}$ & $\begin{array}{l}62.4 \\
54.0 \\
52.2\end{array}$ & $\begin{array}{r}89 \\
105 \\
107\end{array}$ \\
\hline \multicolumn{11}{|c|}{ Emphysema II } \\
\hline D. C. & $\begin{array}{l}\text { Room air } \\
3 \% \mathrm{CO}_{2} \\
4 \% \mathrm{CO}_{2}\end{array}$ & $\begin{array}{l}28 \text { min. } \\
28 \mathrm{~min} .\end{array}$ & $\begin{array}{l}23.0 \\
24.7 \\
28.3\end{array}$ & $\begin{array}{l}0.76 \\
0.74 \\
0.83\end{array}$ & $\begin{array}{l}146 \\
159 \\
161\end{array}$ & $\begin{array}{l}403 \\
696 \\
868\end{array}$ & $\begin{array}{r}9.27 \\
17.20 \\
24.60\end{array}$ & $\begin{array}{l}224 \\
272 \\
267\end{array}$ & $\begin{array}{l}55.7 \\
39 \\
30.7\end{array}$ & $\begin{array}{l}102 \\
110 \\
130\end{array}$ \\
\hline G. B. & $\begin{array}{l}\text { Room air } \\
3 \% \mathrm{CO}_{2} \\
5 \% \mathrm{CO}_{2}\end{array}$ & $\begin{array}{l}28 \text { min. } \\
28 \mathrm{~min} .\end{array}$ & $\begin{array}{l}23.7 \\
25.3 \\
26\end{array}$ & $\begin{array}{l}0.80 \\
0.91 \\
0.84\end{array}$ & $\begin{array}{l}166 \\
147 \\
178\end{array}$ & $\begin{array}{l}453 \\
662 \\
975\end{array}$ & $\begin{array}{l}10.73 \\
16.76 \\
25.30\end{array}$ & $\begin{array}{l}281 \\
347 \\
396\end{array}$ & $\begin{array}{l}61.8 \\
52.5 \\
40.7\end{array}$ & $\begin{array}{r}99 \\
120 \\
125\end{array}$ \\
\hline R. F. & $\begin{array}{l}\text { Room air } \\
3 \% \mathrm{CO}_{2} \\
5 \% \mathrm{CO}_{2}\end{array}$ & $\begin{array}{l}30 \text { min. } \\
28 \mathrm{~min} .\end{array}$ & $\begin{array}{l}22.3 \\
27.7 \\
32\end{array}$ & $\begin{array}{l}0.77 \\
0.75 \\
0.81\end{array}$ & $\begin{array}{l}134 \\
130 \\
117\end{array}$ & $\begin{array}{l}337 \\
513 \\
722\end{array}$ & $\begin{array}{r}7.51 \\
14.23 \\
23.10\end{array}$ & $\begin{array}{l}158 \\
202 \\
213\end{array}$ & $\begin{array}{l}47.1 \\
39.3 \\
29.6\end{array}$ & $\begin{array}{l}104 \\
124 \\
131\end{array}$ \\
\hline A. $\mathrm{H}$. & $\begin{array}{l}\text { Room air } \\
3 \% \mathrm{CO}_{2} \\
5 \% \mathrm{CO}_{2}\end{array}$ & $\begin{array}{l}29 \text { min. } \\
25 \text { min. }\end{array}$ & $\begin{array}{l}16 \\
23.7 \\
25.3\end{array}$ & $\begin{array}{l}0.75 \\
0.75 \\
0.78\end{array}$ & $\begin{array}{l}132 \\
138 \\
162\end{array}$ & $\begin{array}{r}519 \\
694 \\
1,054\end{array}$ & $\begin{array}{r}8.30 \\
16.45 \\
26.68\end{array}$ & $\begin{array}{l}764 \\
338 \\
431\end{array}$ & $\begin{array}{l}50.9 \\
48.6 \\
40.8\end{array}$ & $\begin{array}{r}98 \\
117 \\
123\end{array}$ \\
\hline
\end{tabular}

tients was higher than the average figures for the normal group by $19 \mathrm{ml}$. Although the changes in physiological dead space with $\mathrm{CO}_{2}$ inhalation were approximately the same as the normal percentagewise, the average personal dead space at rest of $208 \mathrm{ml}$. was twice the normal, and the absolute changes were almost twice as great. The alveolar oxygen tensions were consistently lower at rest than in the normal group, with a lesser degree of change during $\mathrm{CO}_{2}$ inhalation.

The relationships between effective alveolar ventilation and arterial blood $\mathrm{CO}_{2}$ tensions and hy- 
drogen ion concentration are found in Table $X$. tion per square meter body surface area at rest The data were handled in the same way as in the normal group, and the stimulus response relationships obtained were found to be linear. Although the age groups are not comparable, it may be noted that the average effective alveolar ventila(1.84 liters) in the Group III and IV emphysema categories was slightly lower than that of 1.93 liters for the normal group. Thus the elevated arterial $\mathrm{CO}_{2}$ tension at rest in the emphysematous groups would presumably stem from the effects of

TABLE IX

Physiologic data relative to the arterial blood at rest and during $\mathrm{CO}_{2}$ inhalation in thirteen emphysematous subjects

\begin{tabular}{|c|c|c|c|c|c|c|c|c|}
\hline Subject & $\begin{array}{l}\text { Inspired } \\
\text { gas } \\
\text { mixture }\end{array}$ & pHs & $\left(\mathbf{H}^{+}\right) \mathbf{a}$ & $\mathrm{Cs}_{\mathrm{OO}_{2}}$ & $\mathrm{~Pa}_{\mathrm{CO}_{2}}$ & $\mathrm{Sa}_{\mathrm{O}_{2}}$ & Vo & $\underset{\left(\mathrm{BB}_{+}\right) \mathrm{b}}{\text { Calc. }}$ \\
\hline \multicolumn{9}{|c|}{ Emphysema IV } \\
\hline A. M. & $\begin{array}{l}\text { Room air } \\
3 \% \mathrm{CO}_{2} \\
5 \% \mathrm{CO}_{2}\end{array}$ & $\begin{array}{l}7.33 \\
7.30 \\
7.28\end{array}$ & $\begin{array}{l}46.8 \\
50.1 \\
52.5\end{array}$ & $\begin{array}{l}65.3 \\
65.3 \\
69.0\end{array}$ & $\begin{array}{l}54 \\
58 \\
64\end{array}$ & $\begin{array}{l}59 \\
77 \\
77\end{array}$ & $\begin{array}{l}47 \\
50 \\
55\end{array}$ & $\begin{array}{l}49 \\
49 \\
49\end{array}$ \\
\hline F. J. & $\begin{array}{l}\text { Room air } \\
3 \% \mathrm{CO}_{2} \\
5 \% \mathrm{CO}_{2}\end{array}$ & $\begin{array}{l}7.41 \\
7.35 \\
7.33\end{array}$ & $\begin{array}{l}38.9 \\
44.7 \\
46.8\end{array}$ & $\begin{array}{l}64.0 \\
65.4 \\
65.2\end{array}$ & $\begin{array}{l}44 \\
52 \\
54\end{array}$ & $\begin{array}{l}79 \\
87 \\
87\end{array}$ & $\begin{array}{l}48 \\
48 \\
49\end{array}$ & $\begin{array}{l}51 \\
50 \\
49\end{array}$ \\
\hline W. D. & $\begin{array}{l}\text { Room air } \\
3 \% \mathrm{CO}_{2} \\
5 \% \mathrm{CO}_{2}\end{array}$ & $\begin{array}{l}7.46 \\
7.45 \\
7.43\end{array}$ & $\begin{array}{l}34.7 \\
35.5 \\
37.2\end{array}$ & $\begin{array}{l}72.6 \\
78.6 \\
79.7\end{array}$ & $\begin{array}{l}45 \\
50 \\
53\end{array}$ & $\begin{array}{l}88 \\
91 \\
91\end{array}$ & $\begin{array}{l}55 \\
55 \\
56\end{array}$ & $\begin{array}{l}56 \\
58 \\
58\end{array}$ \\
\hline R. C. & $\begin{array}{l}\text { Room air } \\
3 \% \mathrm{CO}_{2} \\
5 \% \mathrm{CO}_{2}\end{array}$ & $\begin{array}{l}7.44 \\
7.39 \\
7.35\end{array}$ & $\begin{array}{l}36.6 \\
41.0 \\
44.9\end{array}$ & $\begin{array}{l}76.0 \\
78.9 \\
80.5\end{array}$ & $\begin{array}{l}49.5 \\
57.5 \\
64\end{array}$ & $\begin{array}{l}86 \\
92 \\
92\end{array}$ & $\begin{array}{l}41 \\
42 \\
45\end{array}$ & $\begin{array}{l}\mathbf{5 5} \\
\mathbf{5 5} \\
\mathbf{5 5}\end{array}$ \\
\hline J. R. & $\begin{array}{l}\text { Room air } \\
3 \% \mathrm{CO}_{2} \\
5 \% \mathrm{CO}_{2}\end{array}$ & $\begin{array}{l}7.38 \\
7.36 \\
7.34\end{array}$ & $\begin{array}{l}41.6 \\
44.0 \\
45.7\end{array}$ & $\begin{array}{l}79.8 \\
81.8 \\
81.8\end{array}$ & $\begin{array}{l}59 \\
64 \\
67\end{array}$ & $\begin{array}{l}82 \\
89 \\
92\end{array}$ & $\begin{array}{l}52 \\
51 \\
52\end{array}$ & $\begin{array}{l}55 \\
56 \\
56\end{array}$ \\
\hline C. $\mathbf{M}$. & $\begin{array}{l}\text { Room air } \\
3 \% \mathrm{CO}_{2} \\
5 \% \mathrm{CO}_{2}\end{array}$ & $\begin{array}{l}7.36 \\
7.32 \\
7.30\end{array}$ & $\begin{array}{l}43.7 \\
47.8 \\
50.1\end{array}$ & $\begin{array}{l}71.4 \\
72.0 \\
73.5\end{array}$ & $\begin{array}{l}55 \\
61 \\
65\end{array}$ & $\begin{array}{l}88 \\
94 \\
95\end{array}$ & $\begin{array}{l}60 \\
60 \\
59\end{array}$ & $\begin{array}{l}52 \\
52 \\
52\end{array}$ \\
\hline \multicolumn{9}{|c|}{ Emphyșema III } \\
\hline G. $\mathbf{R}$. & $\begin{array}{l}\text { Room air } \\
3 \% \mathrm{CO}_{2} \\
5 \% \mathrm{CO}_{2}\end{array}$ & $\begin{array}{l}7.42 \\
7.39 \\
7.37\end{array}$ & $\begin{array}{l}38.0 \\
40.7 \\
42.7\end{array}$ & $\begin{array}{l}69.0 \\
68.4 \\
70.2\end{array}$ & $\begin{array}{l}47 \\
50 \\
53\end{array}$ & $\begin{array}{l}88 \\
95 \\
-\end{array}$ & $\begin{array}{l}41 \\
41 \\
42\end{array}$ & $\begin{array}{l}53 \\
52 \\
51\end{array}$ \\
\hline R. D. & $\begin{array}{l}\text { Room air } \\
3 \% \mathrm{CO}_{2} \\
5 \% \mathrm{CO}_{2}\end{array}$ & $\begin{array}{l}7.39 \\
7.36 \\
7.34\end{array}$ & $\begin{array}{l}40.9 \\
43.3 \\
45.8\end{array}$ & $\begin{array}{l}66.1 \\
67.3 \\
68.8\end{array}$ & $\begin{array}{l}48 \\
52 \\
56\end{array}$ & $\begin{array}{l}90 \\
95 \\
94\end{array}$ & $\begin{array}{l}40 \\
40 \\
41\end{array}$ & $\begin{array}{l}52 \\
50 \\
50\end{array}$ \\
\hline J. Y. & $\begin{array}{l}\text { Room air } \\
3 \% \mathrm{CO}_{2} \\
5 \% \mathrm{CO}_{2}\end{array}$ & $\begin{array}{l}7.37 \\
7.36 \\
7.29\end{array}$ & $\begin{array}{l}42.6 \\
43.7 \\
51.2\end{array}$ & $\begin{array}{l}66.1 \\
68.6 \\
68.5\end{array}$ & $\begin{array}{l}50 \\
53 \\
62\end{array}$ & $\begin{array}{l}87 \\
94 \\
99\end{array}$ & $\begin{array}{l}48 \\
48 \\
48\end{array}$ & $\begin{array}{l}\mathbf{5 1} \\
\mathbf{5 1} \\
\mathbf{5 0}\end{array}$ \\
\hline \multicolumn{9}{|c|}{ Emphysema II } \\
\hline D. C. & $\begin{array}{l}\text { Room air } \\
3 \% \mathrm{CO}_{2} \\
5 \% \mathrm{CO}_{2}\end{array}$ & $\begin{array}{l}7.46 \\
7.45 \\
7.43\end{array}$ & $\begin{array}{l}34.7 \\
35.5 \\
37.2\end{array}$ & $\begin{array}{l}61.1 \\
61.1 \\
62.0\end{array}$ & $\begin{array}{l}38 \\
39 \\
41\end{array}$ & $\begin{array}{l}90 \\
91 \\
95\end{array}$ & $\begin{array}{l}41 \\
41 \\
42\end{array}$ & $\begin{array}{l}50 \\
50 \\
50\end{array}$ \\
\hline G. B. & $\begin{array}{l}\text { Room air } \\
3 \% \mathrm{CO}_{2} \\
5 \% \mathrm{CO}_{2}\end{array}$ & $\begin{array}{l}7.40 \\
7.39 \\
7.37\end{array}$ & $\begin{array}{l}39.8 \\
40.7 \\
42.7\end{array}$ & $\begin{array}{l}58.0 \\
60.0 \\
61.4\end{array}$ & $\begin{array}{l}41 \\
43 \\
46\end{array}$ & $\begin{array}{l}89 \\
91 \\
97\end{array}$ & $\begin{array}{l}46 \\
47 \\
47\end{array}$ & $\begin{array}{l}48 \\
49 \\
49\end{array}$ \\
\hline R. F. & $\begin{array}{l}\text { Room air } \\
3 \% \mathrm{CO}_{2} \\
5 \% \mathrm{CO}_{2}\end{array}$ & $\begin{array}{l}7.40 \\
7.39 \\
7.33\end{array}$ & $\begin{array}{l}40.1 \\
40.9 \\
46.6\end{array}$ & $\begin{array}{l}50.3 \\
51.8 \\
51.8\end{array}$ & $\begin{array}{l}36 \\
37.5 \\
42\end{array}$ & $\begin{array}{l}96 \\
96 \\
95\end{array}$ & $\begin{array}{l}39 \\
40 \\
42\end{array}$ & $\begin{array}{l}45 \\
45 \\
44\end{array}$ \\
\hline A. $\mathbf{H}$. & $\begin{array}{l}\text { Room air } \\
3 \% \mathrm{CO}_{2} \\
5 \% \mathrm{CO}_{2}\end{array}$ & $\begin{array}{l}7.44 \\
7.43 \\
7.38\end{array}$ & $\begin{array}{l}36.3 \\
37.2 \\
41.7\end{array}$ & $\begin{array}{l}62.4 \\
62.2 \\
63.9\end{array}$ & $\begin{array}{l}40 \\
41.5 \\
47\end{array}$ & $\begin{array}{l}92 \\
95 \\
96\end{array}$ & $\begin{array}{l}43 \\
44 \\
47\end{array}$ & $\begin{array}{l}50 \\
50 \\
50\end{array}$ \\
\hline
\end{tabular}


TABLE $x$

Physiologic data relative to effective aloeolas acutilation and sensitivity to the carbon dioxide-hydrogen ion stivinulus in thirtecin emplhysematous subjects

\begin{tabular}{|c|c|c|c|c|c|c|c|c|}
\hline Subject & $\begin{array}{l}\text { Inspired } \\
\text { mas } \\
\text { mixture }\end{array}$ & $\dot{\mathbf{v}}_{\mathbf{\Lambda}}$ & $\dot{\mathbf{V} N \mathbf{M}}$ & VR & $\frac{\mathrm{Pa}_{\mathrm{OO}_{2}}}{\mathrm{VR}}$ & $\frac{\dot{v}_{\Delta / M_{2}}}{\mathrm{P}_{\mathrm{DOO}_{2}}}$ & $\frac{\left(\mathrm{H}^{+}\right) \mathbf{a}}{\mathrm{VR}}$ & $\frac{\nabla_{\Lambda} / M^{2}}{\left(H^{+}\right)_{2}}$ \\
\hline \multicolumn{9}{|c|}{ Emphysema IV } \\
\hline A. $\mathbf{R}$. & $\begin{array}{l}\text { Room air } \\
3 \% \mathrm{CO}_{2} \\
5 \% \mathrm{CO}_{2}\end{array}$ & $\begin{array}{l}2.68 \\
3.99 \\
5.78\end{array}$ & $\begin{array}{l}1.62 \\
2.42 \\
3.50\end{array}$ & $\begin{array}{l}1 \\
1.49 \\
2.15\end{array}$ & 8.6 & 0.2 & 5.7 & 0.3 \\
\hline F. J. & $\begin{array}{l}\text { Room air } \\
3 \% \mathrm{CO}_{2} \\
5 \% \mathrm{CO}_{2}\end{array}$ & $\begin{array}{l}3.18 \\
5.02 \\
5.82\end{array}$ & $\begin{array}{l}1.82 \\
2.87 \\
3.33\end{array}$ & $\begin{array}{l}1 \\
1.57 \\
1.83\end{array}$ & 13.0 & 0.1 & 9.6 & 0.2 \\
\hline W. D. & $\begin{array}{l}\text { Room air } \\
3 \% \mathrm{CO}_{2} \\
5 \% \mathrm{CO}_{2}\end{array}$ & $\begin{array}{l}3.91 \\
6.32 \\
8.16\end{array}$ & $\begin{array}{l}2.38 \\
3.85 \\
4.98\end{array}$ & $\begin{array}{l}1 \\
1.61 \\
2.08\end{array}$ & 7.4 & 0.3 & 2.3 & 1.1 \\
\hline R. C. & $\begin{array}{l}\text { Room air } \\
3 \% \mathrm{CO}_{2} \\
5 \% \mathrm{CO}_{2}\end{array}$ & $\begin{array}{l}3.29 \\
4.70 \\
5.98\end{array}$ & $\begin{array}{l}1.82 \\
2.60 \\
3.30\end{array}$ & $\begin{array}{l}1 \\
1.43 \\
1.81\end{array}$ & 17.0 & 0.1 & 10.1 & 0.2 \\
\hline J. R. & $\begin{array}{l}\text { Room air } \\
3 \% \mathrm{CO}_{2} \\
5 \% \mathrm{CO}_{2}\end{array}$ & $\begin{array}{l}2.85 \\
3.83 \\
4.88\end{array}$ & $\begin{array}{l}1.59 \\
2.14 \\
2.72\end{array}$ & $\begin{array}{l}1 \\
1.34 \\
1.71\end{array}$ & 12.0 & 0.1 & 6.7 & 0.3 \\
\hline C. $\mathbf{M}$. & $\begin{array}{l}\text { Room air } \\
3 \% \mathrm{CO}_{2} \\
5 \% \mathrm{CO}_{2}\end{array}$ & $\begin{array}{l}2.60 \\
3.33 \\
4.55\end{array}$ & $\begin{array}{l}1.70 \\
2.18 \\
2.97\end{array}$ & $\begin{array}{l}1 \\
1.28 \\
1.75\end{array}$ & 14.0 & 0.1 & 9.7 & 0.2 \\
\hline \multicolumn{9}{|c|}{ Emphysema III } \\
\hline G. R. & $\begin{array}{l}\text { Room air } \\
3 \% \mathrm{CO}_{2} \\
5 \% \mathrm{CO}_{2}\end{array}$ & $\begin{array}{l}3.48 \\
5.38 \\
9.60\end{array}$ & $\begin{array}{l}1.91 \\
2.96 \\
5.27\end{array}$ & $\begin{array}{l}1 \\
1.55 \\
2.75\end{array}$ & 4.4 & 0.5 & 2.9 & 0.6 \\
\hline R. D. & $\begin{array}{l}\text { Room air } \\
3 \% \mathrm{CO}_{2} \\
5 \% \mathrm{CO}_{2}\end{array}$ & $\begin{array}{l}2.72 \\
4.66 \\
5.90\end{array}$ & $\begin{array}{l}1.77 \\
3.03 \\
3.83\end{array}$ & $\begin{array}{l}1 \\
1.71 \\
2.17\end{array}$ & 6.5 & 0.3 & 4.0 & 0.5 \\
\hline J. Y. & $\begin{array}{l}\text { Room air } \\
3 \% \mathrm{CO}_{2} \\
5 \% \mathrm{CO}_{2}\end{array}$ & $\begin{array}{l}3.50 \\
5.52 \\
8.04\end{array}$ & $\begin{array}{l}1.91 \\
3.02 \\
4.39\end{array}$ & $\begin{array}{l}1 \\
1.58 \\
2.30\end{array}$ & 8.4 & 0.2 & 5.0 & 0.4 \\
\hline \multicolumn{9}{|c|}{ Emphysema II } \\
\hline D. C. & $\begin{array}{l}\text { Room air } \\
3 \% \mathrm{CO}_{2} \\
5 \% \mathrm{CO}_{2}\end{array}$ & $\begin{array}{r}4.11 \\
10.33 \\
17.02\end{array}$ & $\begin{array}{r}2.55 \\
6.42 \\
10.57\end{array}$ & $\begin{array}{l}1 \\
2.52 \\
4.14\end{array}$ & 0.9 & 2.9 & 0.8 & 3.4 \\
\hline G. B. & $\begin{array}{l}\text { Room air } \\
3 \% \mathrm{CO}_{2} \\
5 \% \mathrm{CO}_{2}\end{array}$ & $\begin{array}{r}4.10 \\
7.98 \\
15.00\end{array}$ & $\begin{array}{r}2.81 \\
5.46 \\
10.28\end{array}$ & $\begin{array}{l}1 \\
1.94 \\
3.67\end{array}$ & 1.9 & 1.5 & 1.1 & 2.6 \\
\hline R.F. & $\begin{array}{l}\text { Room air } \\
3 \% \mathrm{CO}_{2} \\
5 \% \mathrm{CO}_{2}\end{array}$ & $\begin{array}{r}3.97 \\
8.66 \\
16.25\end{array}$ & $\begin{array}{r}2.48 \\
5.41 \\
10.17\end{array}$ & $\begin{array}{l}1 \\
2.18 \\
4.10\end{array}$ & 1.7 & 1.3 & 1.5 & 1.6 \\
\hline A. $\mathrm{H}$. & $\begin{array}{l}\text { Room air } \\
3 \% \mathrm{CO}_{2} \\
5 \% \mathrm{CO}_{2}\end{array}$ & $\begin{array}{r}4.07 \\
8.45 \\
15.80\end{array}$ & $\begin{array}{l}2.15 \\
4.47 \\
8.37\end{array}$ & $\begin{array}{l}1 \\
2.08 \\
3.88\end{array}$ & 2.1 & 1.0 & 1.8 & 1.2 \\
\hline
\end{tabular}

diminished effective alveolar ventilation and some slight increase in $\mathrm{CO}_{2}$ production, possibly related to greater respiratory work. The average percentage increases in effective alveolar ventilation with 3 per cent and 5 per cent $\mathrm{CO}_{2}$ inhalation were less than half the average normal values. To double the effective alveolar ventilation, which was accomplished in only five of the nine cases, an increase of 4.4 to $17 \mathrm{~mm}$. $\mathrm{Hg}$ in the arterial $\mathrm{CO}_{2}$ tension was required. The changes were well out of the normal range in all instances, usually several times the average normal value. The as- 
sociated change in hydrogen ion concentration necessary to double the effective alveolar ventilation was usually three or more times the average normal value, although in two instances it fell at the upper limit of the normal range. For each $\mathrm{mm} . \mathrm{Hg}$ rise in the arterial $\mathrm{CO}_{2}$ tension there was an increase in effective alveolar ventilation per square meter body surface area of only 0.1 to 0.5 liter per minute, as compared with an average increase of 1.2 liters per minute in the normal group. The smallest increase in the normal group was 0.8 liter per minute. For a billionth of a mole per liter increase in arterial blood hydrogen ion concentration, the effective alveolar ventilation per square meter body surface area rose only 0.2 to 1.1 liters per minute. The average value in the normal group was 1.9 liters per minute, and in only one instance was the value of a Group III or IV emphysema patient within the normal range.

Observations on four emphysematous patients without $\mathrm{CO}_{2}$ retention (Group II) are shown in Tables VIII, IX and X. The findings at rest and changes with $\mathrm{CO}_{2}$ inhalation are roughly comparable to the normal with regard to respiratory exchange ratio, respiratory frequency, oxygen consumption, tidal volume, total pulmonary ventilation, and calculated mean effective alveolar oxygen tension. The physiological dead space at rest is greater than normal as would be expected. Except for lowered oxygen saturation, the results of arterial blood analyses differ little from those described in the normal group. Effective alveolar ventilation per square meter body surface area at rest was greater than the average normal value in all instances. Nevertheless the changes in effective alveolar ventilation with $\mathrm{CO}_{2}$ inhalation were essentially the same as in the normal group, i.e., roughly two times the resting value with 3 per cent $\mathrm{CO}_{2}$ inhalation, and four times the resting value with 5 per cent $\mathrm{CO}_{2}$. Relationships between effective alveolar ventilation and arterial $\mathrm{CO}_{2}$ tension or hydrogen ion concentration obtained in the manner previously described, fell within the normal range, indicating a normal ventilatory response to the carbon dioxide-hydrogen ion stimulus.

\section{Cyanotic congenital heart disease}

Of the patients with cyanotic congenital heart disease, the diagnosis of pulmonic stenosis and interatrial defect had been made in one, and that of Eisenmenger's Complex in two, on the basis of cardiac catheterization studies. Spirometric data and clinical notes on these patients are shown in Table XII. Vital and maximum breathing capacities were within the normal range except in one instance where moderate kyphosis probably accounted for some reduction in the vital capacity. Observations on ventilation and arterial blood at rest and during $\mathrm{CO}_{2}$ inhalation appear in Tables XIII, XIV, and XV. All three patients had arterial oxygen unsaturation and polycythemia of appreciable degree. In addition two had lowered arterial $\mathrm{CO}_{2}$ tensions and a tendency to alkalosis. Oxygen consumption per square meter body surface area, total pulmonary ventilation, and ef-

TABLE XI

Response of emphysematous subjects (groups III and IV) and normal subjects to $\mathrm{CO}_{2}$ inhalation. Comparison of sensitivity to the carbon dioxide-hydrogen ion stimulus

\begin{tabular}{|c|c|c|c|c|c|c|c|c|c|c|}
\hline \multirow[b]{2}{*}{$\begin{array}{c}\text { Inspired gas } \\
\text { mixture }\end{array}$} & \multicolumn{2}{|c|}{$\begin{array}{l}\text { pH average } \\
\text { values }\end{array}$} & \multicolumn{2}{|c|}{$\begin{array}{l}\mathrm{Pa}_{\mathrm{CO}_{2}} \text { average } \\
\text { increase }\end{array}$} & \multicolumn{2}{|c|}{$\begin{array}{l}\mathrm{PA}_{2} \text { average } \\
\text { values }\end{array}$} & \multicolumn{2}{|c|}{$\begin{array}{l}\dot{\mathbf{V}}_{\mathbf{R}} \text { average } \\
\text { increase }\end{array}$} & \multicolumn{2}{|c|}{$\begin{array}{l}\dot{\mathbf{V}}_{\text {A average }} \\
\text { increase }\end{array}$} \\
\hline & Normal & $\underset{\text { sema }}{\text { Emphy- }}$ & Normal & $\begin{array}{c}\text { Emphy- } \\
\text { sema }\end{array}$ & Normal & $\begin{array}{c}\text { Emphy- } \\
\text { sema }\end{array}$ & Normal & $\begin{array}{c}\text { Emphy- } \\
\text { sema }\end{array}$ & Normal & $\underset{\text { Eema }}{\text { Emphy- }}$ \\
\hline \multirow{3}{*}{$\begin{array}{l}\text { Room air } \\
3 \% \mathrm{CO}_{2} \text { in air } \\
5 \% \mathrm{CO}_{2} \text { in air }\end{array}$} & \multirow[b]{2}{*}{$\begin{array}{l}7.42 \\
7.41 \\
7.38\end{array}$} & \multirow[b]{2}{*}{$\begin{array}{l}7.40 \\
7.36 \\
7.34\end{array}$} & \multicolumn{2}{|c|}{$\mathrm{mm} . \mathrm{Hg}_{\mathrm{g}}$} & \multicolumn{2}{|c|}{ mm. $H_{8}$} & \multicolumn{2}{|c|}{$\%$} & \multicolumn{2}{|c|}{$\%$} \\
\hline & & & $\begin{array}{l}\overline{2.5} \\
5.0\end{array}$ & $\begin{array}{l}\overline{5.1} \\
9.6\end{array}$ & $\begin{array}{l}97 \\
117 \\
125\end{array}$ & $\begin{array}{r}86 \\
102 \\
107\end{array}$ & $\begin{array}{l}\overline{202} \\
329\end{array}$ & $\begin{array}{l}\overline{144} \\
192\end{array}$ & $\begin{array}{l}\overline{222} \\
382\end{array}$ & $\begin{array}{l}\overline{151} \\
206\end{array}$ \\
\hline & & & $\mathrm{Pa}_{\mathrm{CO}_{2}} /$ & $\mathbf{V R}$ & $\frac{\dot{V}_{\mathrm{A}} / \mathrm{M}^{2}}{\mathrm{~Pa}_{\mathrm{CO}_{2}}}$ & & $\left(\mathbf{H}^{+}\right) \mathbf{a} / \mathbf{V R}$ & & $\frac{\dot{\mathrm{V}}_{\mathbf{A}} / \mathrm{M}_{\mathbf{2}}}{\left(\mathrm{H}^{+}\right)_{\mathbf{a}}}$ & \\
\hline \multicolumn{3}{|c|}{$\begin{array}{l}\text { Normal average value } \\
\text { Emphysema range }\end{array}$} & \multicolumn{2}{|c|}{$\begin{array}{l}1.7 \\
4.4-17\end{array}$} & $0.1-0.5$ & & $\begin{array}{c}1.3 \\
2.3-10.1\end{array}$ & & $\begin{array}{c}1.9 \\
0.2-1.1\end{array}$ & \\
\hline
\end{tabular}


TABLE XII

Spirometric data and clinical findings in patients with cyanotic congenital heart disease, chronic acidosis, and chronic alkalosis

\begin{tabular}{|c|c|c|c|c|c|c|c|c|c|}
\hline \multicolumn{3}{|c|}{ Patient } & \multirow[b]{2}{*}{ B.S.A. } & \multicolumn{2}{|c|}{ Vital capacity } & \multicolumn{3}{|c|}{ Maximum breathing cap. } & \multirow[b]{2}{*}{ Notes } \\
\hline Sex & 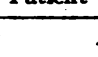 & $\overline{\text { Age }}$ & & $m l$. & $\begin{array}{c}\% \\
\text { pred. }\end{array}$ & L./min. & L. $/ M^{\mathbf{m}}$. $/$ & $\begin{array}{c}\% \\
\text { pred. }\end{array}$ & \\
\hline \multicolumn{10}{|c|}{ Cyanotic congenital heart disease } \\
\hline $\mathbf{M}$ & J.B. & 23 & 1.67 & 4,630 & 108 & 124.5 & 75 & 100 & $\begin{array}{l}\text { Moderate reduction in exercise tolerance. } \\
\text { Pulmonic stenosis with interatrial de- }\end{array}$ \\
\hline $\mathbf{M}$ & R. W. & 28 & 1.64 & 2,170 & 57 & 97.3 & 59.3 & 83 & $\begin{array}{l}\text { Kyphosis of moderate degree. Eisen- } \\
\text { menger's Complex. }\end{array}$ \\
\hline $\mathbf{M}$ & W. M. & & 1.86 & 5,880 & 137 & 170 & 91.3 & 126 & $\begin{array}{l}\text { Moderate reduction in exercise tolerance. } \\
\text { Eisenmenger's Complex. }\end{array}$ \\
\hline \multicolumn{10}{|c|}{ Chronic acidosis } \\
\hline $\mathbf{F}$ & G. $\mathbf{K}$. & 30 & 1.58 & 2,590 & 89 & 72 & 45.6 & 80 & $\begin{array}{l}\text { Chronic glomerulonephritis since age } 5 \text {. } \\
\text { Nitrogen retention documented for } 3 \\
\text { yrs. prior to study. At time of study } \\
\text { serum electrolyte levels, mEq./L., } \mathrm{Na} \\
135.8 \text { a }\end{array}$ \\
\hline $\mathbf{M}$ & J. K. & 48 & 1.67 & 2,146 & 61 & 71.7 & 43 & 70 & $\begin{array}{l}\text { Nephritis since age } 17 \text { with documented } \\
\text { nitrogen retention for } 7 \text { yrs. prior to } \\
\text { study. Lowered venous serum CO} \\
\text { content found on occasion over several } \\
\text { years. At time of study serum electro- } \\
\text { lyte levels, mEq./L., } \mathrm{Na} 131.4, \mathrm{Cl} 104.6 \text {, } \\
\mathrm{K} 5.6, \mathrm{BUN} 96 \mathrm{mg} . \% \text {. }\end{array}$ \\
\hline $\mathbf{M}$ & E. W. & 51 & 1.91 & 2,695 & 80 & 71.5 & 37.4 & 62 & $\begin{array}{l}\text { Documented nitrogen retention for } 3 \text { yrs. } \\
\text { prior to study. Presumed diagnosis } \\
\text { arteriolar nephrosclerosis. At time of } \\
\text { study serum electrolyte levels, mEg./ } \\
\mathrm{L} ., \mathrm{Na} \text { 139, Cl 125, K 7.1, BUN 58 mg. \%. }\end{array}$ \\
\hline \multicolumn{10}{|c|}{ Chronic alkalosis } \\
\hline $\mathbf{F}$ & K. S. & 49 & 1.65 & 2,030 & 80 & 57.3 & 35 & 73 & 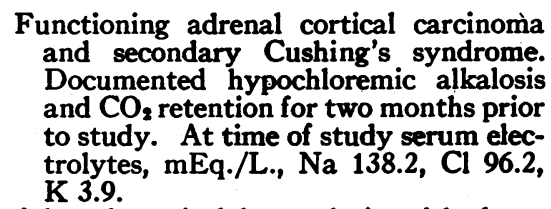 \\
\hline $\mathbf{F}$ & J. T. & 41 & 1.59 & 4,330 & 152 & 153.4 & 96.5 & 187 & $\begin{array}{l}\text { Adrenal cortical hyperplasia with docu- } \\
\text { mented hypochloremic alkalosis for two } \\
\text { months prior to therapy. No } \mathrm{CO}_{2} \text { re- } \\
\text { tention for approx. } 4 \text { mos. at the time } \\
\text { of study. }\end{array}$ \\
\hline
\end{tabular}

fective alveolar ventilation ${ }^{6}$ at rest were uniformly elevated. The effective alveolar ventilation per

- The effective alveolar ventilation in these cases, as in the normal group, was calculated on the assumption that alveolar $\mathrm{CO}_{2}$ tension was equivalent to arterial $\mathrm{CO}_{2}$ tension. This of course is not a valid assumption in these patients because of venous admixture to left ventricular blood through the right to left intracardiac shunt. Thus the actual effective alveolar ventilation at rest was somewhat higher than that recorded in Table XV. Since no pulmonary arterial blood samples were obtained at the time of the $\mathrm{CO}_{2}$ inhalation study which would allow estimation of the degree of venous admixture, no calculations were carried out attempting to correct arterial $\mathrm{CO}_{2}$ tension to alveolar $\mathrm{CO}_{2}$ tension. This would square meter body surface area was 50 per cent or more greater than the average normal value in all three patients. With $\mathrm{CO}_{2}$ inhalation, the changes

introduce no error in the slope of the $\mathrm{CO}_{2}$ response curve if the degree of shunt remained constant with $\mathrm{CO}_{2}$ inhalation. However, even if the amount shunted were to change by as much as 10 per cent with $\mathrm{CO}_{2}$ inhalation, the difference in the corrected alveolar $\mathrm{CO}_{2}$ tension would be no greater than $1 \mathrm{~mm}$. $\mathrm{Hg}$. Thus, no appreciable error has been introduced into the measurement of the change in effective alveolar ventilation with $\mathrm{CO}_{2}$ inhalation, a partial determinant of the slope of the stimulus response curve. Because of the lack of specific values for alveolar $\mathrm{CO}_{2}$ tension, no calculations of mean effective alveolar oxygen tension were carried out. 
in respiratory exchange ratio and frequency, tidal volume, ventilation, physiological dead space, $\mathrm{pH}$, $\mathrm{CO}_{2}$ tension, and buffer base were similar to those observed in the normal group. However, the increase in oxygen consumption per square meter body surface area with 5 per cent $\mathrm{CO}_{2}$ breathing in these patients was consistent, and was presumably related to the high rates of ventilation observed. As expected, only a small increase in arterial oxygen saturation occurred with $\mathrm{CO}_{2}$ inhalation, despite substantial increments in effective alveolar ventilation. As in the normal group, the relationships between changes in effective alveolar ventilation and arterial $\mathrm{CO}_{2}$ tension or hydrogen ion concentration were found to be essentially linear. The observed changes in arterial $\mathrm{CO}_{2}$ tension and hydrogen ion concentration associated with doubling of the effective alveolar ventilation fell within the normal range, although an increased response in terms of effective alveolar ventilation per square meter of body surface area occurred in one instance.

\section{Chronic metabolic acidosis}

Three patients with uremia and chronic metabolic acidosis secondary to renal failure were studied. Clinical findings, blood electrolyte levels at the time of study, and spirometric data appear in Table XII.

All showed a tendency to reduction in maximum breathing capacity which might well have been related to the presence of weakness and easy fa-

TABLE XIII

Physiologic data relative to pulmonary ventilation at rest and during $\mathrm{CO}_{2}$ inhalation in patients with cyanotic congenital heart disease, chronic acidosis, and chronic alkalosis

\begin{tabular}{|c|c|c|c|c|c|c|c|c|c|c|}
\hline \multirow[b]{2}{*}{ Patient } & \multirow{2}{*}{$\begin{array}{l}\text { Inspired } \\
\text { gas } \\
\text { mixture }\end{array}$} & \multirow{2}{*}{$\begin{array}{c}\text { Time on } \\
\text { mixture } \\
\text { (min.) }\end{array}$} & \multirow[b]{2}{*}{ f } & \multirow[b]{2}{*}{$\mathbf{R s}$} & \multirow[b]{2}{*}{$\dot{\mathrm{v}}_{\mathrm{O}_{2}} / \mathrm{M}^{2}$} & \multirow[b]{2}{*}{$\mathbf{V} \mathbf{T}$} & \multirow[b]{2}{*}{$\dot{\mathbf{V}}_{\mathbf{B}}$} & \multicolumn{2}{|c|}{ VD } & \multirow{2}{*}{$\begin{array}{l}\text { Calc. } \\
\mathrm{PA}_{\mathrm{O}_{2}}\end{array}$} \\
\hline & & & & & & & & $m l$. & $\% \dot{\mathrm{V}_{E}}$ & \\
\hline \multicolumn{11}{|c|}{ Cyanotic congenital heart disease ${ }^{*}$} \\
\hline J. B. & $\begin{array}{l}\text { Room air } \\
3 \% \mathrm{CO}_{2} \\
4 \% \mathrm{CO}_{2}\end{array}$ & $\begin{array}{l}-\overline{26} \\
30\end{array}$ & $\begin{array}{l}18 \\
20.3 \\
25\end{array}$ & $\begin{array}{l}0.76 \\
0.88 \\
0.71\end{array}$ & $\begin{array}{l}148 \\
158 \\
206\end{array}$ & $\begin{array}{r}524 \\
1,265 \\
1,750\end{array}$ & $\begin{array}{r}9.43 \\
25.70 \\
43.70\end{array}$ & $\begin{array}{l}212 \\
187 \\
120\end{array}$ & $\begin{array}{r}40.3 \\
14.7 \\
7.0\end{array}$ & \\
\hline R. W. & $\begin{array}{l}\text { Room air } \\
3 \% \mathrm{CO}_{2} \\
4 \% \mathrm{CO}_{2}\end{array}$ & $\begin{array}{l}-26 \\
29\end{array}$ & $\begin{array}{l}31.7 \\
33.3 \\
36.7\end{array}$ & $\begin{array}{l}0.76 \\
0.71 \\
0.74\end{array}$ & $\begin{array}{l}179 \\
151 \\
200\end{array}$ & $\begin{array}{l}388 \\
666 \\
904\end{array}$ & $\begin{array}{l}12.30 \\
22.15 \\
33.20\end{array}$ & $\begin{array}{l}228 \\
422 \\
477\end{array}$ & $\begin{array}{l}58.7 \\
63.3 \\
52.8\end{array}$ & \\
\hline W. M. & $\begin{array}{l}\text { Room air } \\
3 \% \mathrm{CO}_{2} \\
4 \% \mathrm{CO}_{2}\end{array}$ & $\begin{array}{l}-\overline{27} \\
26\end{array}$ & $\begin{array}{l}16 \\
20.2 \\
23\end{array}$ & $\begin{array}{l}0.75 \\
0.87 \\
0.71\end{array}$ & $\begin{array}{l}162 \\
137 \\
203\end{array}$ & $\begin{array}{r}680 \\
1,362 \\
2,010\end{array}$ & $\begin{array}{l}10.88 \\
27.50 \\
46.15\end{array}$ & $\begin{array}{l}271 \\
604 \\
888\end{array}$ & $\begin{array}{l}39.8 \\
44.3 \\
44.1\end{array}$ & \\
\hline \multicolumn{11}{|c|}{ Chronic acidosis } \\
\hline G. $\mathbf{K}$. & $\begin{array}{l}\text { Room air } \\
3 \% \mathrm{CO}_{2} \\
5 \% \mathrm{CO}_{2}\end{array}$ & $\begin{array}{l}-\overline{27} \\
27\end{array}$ & $\begin{array}{l}15.7 \\
20 \\
20.7\end{array}$ & $\begin{array}{l}0.81 \\
0.82 \\
0.94\end{array}$ & $\begin{array}{l}131 \\
138 \\
139\end{array}$ & $\begin{array}{r}424 \\
717 \\
1,300\end{array}$ & $\begin{array}{r}6.66 \\
14.35 \\
26.90\end{array}$ & $\begin{array}{l}172 \\
258 \\
345\end{array}$ & $\begin{array}{l}40.5 \\
36.1 \\
26.6\end{array}$ & $\begin{array}{l}105 \\
124 \\
131\end{array}$ \\
\hline J. K. & $\begin{array}{l}\text { Room air } \\
3 \% \mathrm{CO}_{2} \\
5 \% \mathrm{CO}_{2}\end{array}$ & $\begin{array}{l}\overline{23} \\
\text { Tolerated } \\
\text { only } 8 \mathrm{~min} .\end{array}$ & $\begin{array}{l}29.7 \\
29.3 \\
49\end{array}$ & $\begin{array}{l}0.80 \\
0.72 \\
-\end{array}$ & $\begin{array}{l}187 \\
232 \\
-\end{array}$ & $\begin{array}{l}387 \\
773 \\
992\end{array}$ & $\begin{array}{l}11.48 \\
22.65 \\
48.60\end{array}$ & $\begin{array}{l}211 \\
359 \\
-\end{array}$ & $\begin{array}{c}54.5 \\
46.4 \\
-\end{array}$ & $\begin{array}{l}101 \\
119 \\
-\end{array}$ \\
\hline W. E. & $\begin{array}{l}\text { Room air } \\
3 \% \mathrm{CO}_{2} \\
5 \% \mathrm{CO}_{2}\end{array}$ & $\begin{array}{c}\overline{30} \\
\text { Tolerated } \\
\text { only } 10 \mathrm{~min} .\end{array}$ & $\begin{array}{l}16.7 \\
25.7 \\
26\end{array}$ & $\begin{array}{c}0.82 \\
0.76 \\
-\end{array}$ & $\begin{array}{l}119 \\
136 \\
-\end{array}$ & $\begin{array}{r}514 \\
795 \\
1,345\end{array}$ & $\begin{array}{r}8.59 \\
20.43 \\
34.97\end{array}$ & $\begin{array}{l}231 \\
369 \\
-\end{array}$ & $\begin{array}{c}44.9 \\
46.4 \\
-\end{array}$ & $\begin{array}{l}106 \\
121 \\
-\end{array}$ \\
\hline \multicolumn{11}{|c|}{ Chronic alkalosis } \\
\hline K. S. & $\begin{array}{l}\text { Room air } \\
3 \% \mathrm{CO}_{2} \\
5 \% \mathrm{CO}_{2}\end{array}$ & $\begin{array}{l}\overline{34} \\
30\end{array}$ & $\begin{array}{l}20 \\
23.7 \\
23\end{array}$ & $\begin{array}{l}0.73 \\
0.72 \\
0.81\end{array}$ & $\begin{array}{l}148 \\
136 \\
137\end{array}$ & $\begin{array}{l}320 \\
435 \\
693\end{array}$ & $\begin{array}{r}6.41 \\
10.33 \\
15.93\end{array}$ & $\begin{array}{l}139 \\
182 \\
267\end{array}$ & $\begin{array}{l}43.3 \\
41.8 \\
38.5\end{array}$ & $\begin{array}{r}93 \\
114 \\
120\end{array}$ \\
\hline J. T. & $\begin{array}{l}\text { Room air } \\
3 \% \mathrm{CO}_{2} \\
5 \% \mathrm{CO}_{2}\end{array}$ & $\begin{array}{l}-\overline{26} \\
25\end{array}$ & $\begin{array}{l}8.3 \\
10 \\
14\end{array}$ & $\begin{array}{l}0.72 \\
0.70 \\
0.80\end{array}$ & $\begin{array}{l}145 \\
143 \\
141\end{array}$ & $\begin{array}{r}651 \\
915 \\
1,232\end{array}$ & $\begin{array}{r}5.41 \\
9.15 \\
17.26\end{array}$ & $\begin{array}{l}234 \\
364 \\
610\end{array}$ & $\begin{array}{l}36.0 \\
39.8 \\
49.5\end{array}$ & $\begin{array}{r}95 \\
111 \\
120\end{array}$ \\
\hline
\end{tabular}

* See footnote 6 . 
TABLE XIV

Physiologic data relative to the arterial blood at rest and during $\mathrm{CO}_{2}$ inhalation in patients with cyanotic congenital heart disease, chronic acidosis, and chronic alkalosis

\begin{tabular}{|c|c|c|c|c|c|c|c|c|}
\hline Patient & $\begin{array}{l}\text { Inspired } \\
\text { gass } \\
\text { mixture }\end{array}$ & pHs & $\left(\mathrm{H}^{+}\right) \mathbf{a}$ & $\mathrm{Cs}_{\mathrm{CO}_{2}}$ & $\mathrm{~Pa}_{\mathrm{OO}}$ & Sag, & Vo & $\underset{\left(\mathrm{Ba}_{+}\right) \mathrm{b}}{\text { Calc. }}$ \\
\hline \multicolumn{9}{|c|}{ Cyanotic congenital heart disease } \\
\hline J. B. & $\begin{array}{l}\text { Room air } \\
3 \% \mathrm{CO}_{2} \\
4 \% \mathrm{CO}_{2}\end{array}$ & $\begin{array}{l}7.45 \\
7.44 \\
7.40\end{array}$ & $\begin{array}{l}35.4 \\
36.3 \\
39.8\end{array}$ & $\begin{array}{l}45.7 \\
49.1 \\
49.7\end{array}$ & $\begin{array}{l}29 \\
32 \\
35\end{array}$ & $\begin{array}{l}86 \\
87 \\
88\end{array}$ & $\begin{array}{l}62 \\
62 \\
62\end{array}$ & $\begin{array}{l}48 \\
48 \\
47\end{array}$ \\
\hline R. W. & $\begin{array}{l}\text { Room air } \\
3 \% \mathrm{CO}_{2} \\
4 \% \mathrm{CO}_{2}\end{array}$ & $\begin{array}{l}7.40 \\
7.39 \\
7.36\end{array}$ & $\begin{array}{l}39.8 \\
40.7 \\
43.6\end{array}$ & $\begin{array}{l}53.7 \\
56.0 \\
55.9\end{array}$ & $\begin{array}{l}38.5 \\
41 \\
43\end{array}$ & $\begin{array}{l}83 \\
85 \\
88\end{array}$ & $\begin{array}{l}75 \\
76 \\
77\end{array}$ & $\begin{array}{l}50 \\
49 \\
48\end{array}$ \\
\hline W. M. & $\begin{array}{l}\text { Room air } \\
3 \% \mathrm{CO}_{2} \\
4 \% \mathrm{CO}_{2}\end{array}$ & $\begin{array}{l}7.49 \\
7.42 \\
7.40\end{array}$ & $\begin{array}{l}32.3 \\
38.0 \\
39.8\end{array}$ & $\begin{array}{l}51.4 \\
52.0 \\
54.2\end{array}$ & $\begin{array}{l}30 \\
35 \\
38.5\end{array}$ & $\begin{array}{l}87 \\
89 \\
90\end{array}$ & $\begin{array}{l}61 \\
61 \\
62\end{array}$ & $\begin{array}{l}51 \\
49 \\
49\end{array}$ \\
\hline \multicolumn{9}{|c|}{ Chronic acidosis } \\
\hline G. $\mathbf{K}$. & $\begin{array}{l}\text { Room air } \\
3 \% \mathrm{CO}_{2} \\
5 \% \mathrm{CO}_{2}\end{array}$ & $\begin{array}{l}7.36 \\
7.35 \\
7.30\end{array}$ & $\begin{array}{l}43.6 \\
44.7 \\
50.1\end{array}$ & $\begin{array}{l}47.5 \\
49.0 \\
49.5\end{array}$ & $\begin{array}{l}37 \\
38.5 \\
43\end{array}$ & $\begin{array}{r}95 \\
98 \\
100\end{array}$ & $\begin{array}{l}26 \\
27 \\
28\end{array}$ & $\begin{array}{l}41 \\
41 \\
41\end{array}$ \\
\hline J. K. & $\begin{array}{l}\text { Room air } \\
3 \% \mathrm{CO}_{2} \\
5 \% \mathrm{CO}_{2}\end{array}$ & $\begin{array}{c}7.32 \\
7.32 \\
-\end{array}$ & $\begin{array}{c}47.4 \\
48.3 \\
-\end{array}$ & $\begin{array}{c}50.1 \\
49.3 \\
-\end{array}$ & $\begin{array}{l}41.5 \\
42 \\
-\end{array}$ & $\begin{array}{l}94 \\
96 \\
\end{array}$ & $\begin{array}{l}33 \\
35 \\
-\end{array}$ & $\begin{array}{l}42 \\
42 \\
-\end{array}$ \\
\hline W. E. & $\begin{array}{l}\text { Room air } \\
3 \% \mathrm{CO}_{2} \\
5 \% \mathrm{CO}_{2}\end{array}$ & $\begin{array}{l}7.20 \\
7.19 \\
-\end{array}$ & $\begin{array}{l}63.2 \\
64.6 \\
-\end{array}$ & $\begin{array}{c}32.0 \\
33.1 \\
-\end{array}$ & $\begin{array}{l}34.5 \\
37 \\
-\end{array}$ & $\begin{array}{r}94 \\
100 \\
-\end{array}$ & $\begin{array}{l}26 \\
27 \\
-\end{array}$ & $\begin{array}{l}32 \\
32 \\
-\end{array}$ \\
\hline \multicolumn{9}{|c|}{ Chronic alkalosis } \\
\hline K. S. & $\begin{array}{l}\text { Room air } \\
3 \% \mathrm{CO}_{2} \\
5 \% \mathrm{CO}_{2}\end{array}$ & $\begin{array}{l}7.47 \\
7.45 \\
7.42\end{array}$ & $\begin{array}{l}33.9 \\
35.5 \\
38.0\end{array}$ & $\begin{array}{l}69.2 \\
70.5 \\
73.2\end{array}$ & $\begin{array}{l}42.5 \\
45 \\
50\end{array}$ & $\begin{array}{l}91 \\
96 \\
96\end{array}$ & $\begin{array}{l}38 \\
38 \\
37\end{array}$ & $\begin{array}{l}54 \\
54 \\
54\end{array}$ \\
\hline J.T. & $\begin{array}{l}\text { Room air } \\
3 \% \mathrm{CO}_{2} \\
5 \% \mathrm{CO}_{2}\end{array}$ & $\begin{array}{l}7.40 \\
7.38 \\
7.35\end{array}$ & $\begin{array}{l}39.8 \\
41.7 \\
44.6\end{array}$ & $\begin{array}{l}60.1 \\
63.3 \\
65.1\end{array}$ & $\begin{array}{l}42 \\
47 \\
52\end{array}$ & $\begin{array}{l}94 \\
97 \\
97\end{array}$ & $\begin{array}{l}43 \\
43 \\
44\end{array}$ & $\begin{array}{l}48 \\
49 \\
49\end{array}$ \\
\hline
\end{tabular}

tigability. The lowered vital capacity in patient J. K. was possibly associated with the presence of moderate pulmonary congestion.

Observations on the response of these uremic patients to the $\mathrm{CO}_{2}$ stimulus are found in Tables XIII, XIV, and XV. At rest all had an elevated effective alveolar ventilation per square meter body surface area, reduced arterial $\mathrm{pH}$, and anemia. One patient had an elevated oxygen consumption per square meter body surface area. The other two had a somewhat lowered arterial $\mathrm{CO}_{2}$ tension. Two of the three patients were not able to tolerate the 5 per cent $\mathrm{CO}_{2}$ inspired gas mixture for longer than a few minutes, a finding confirmatory of Peabody's experience (19). In these two instances the ventilation measured near the end of this short period on 5 per cent $\mathrm{CO}_{2}$ was 50 per cent or more of the maximum breathing capacity, suggesting that intolerance may result when the ventilatory drive exceeds the patient's physical capacity to respond. The sensitivity to the carbon dioxide-hydrogen ion stimulus in these two cases has been determined, therefore, on the basis of only two points on the stimulus response curve. There was a deficit in the calculated buffer base, as found by Yeomans and Stueck (20) in their uremic subjects, and no change in buffer base occurred with $\mathrm{CO}_{2}$ inhalation.

It can be seen that the response to the carbon dioxide-hydrogen ion stimulus when evaluated in terms of either the alveolar ventilation ratio or effective alveolar ventilation per square meter body surface area fell within the normal range in two patients, but was distinctly increased in a third (J. K.).

\section{Chronic metabolic alkalosis}

Clinical notes on the two patients with chronic metabolic alkalosis and $\mathrm{CO}_{2}$ retention secondary 
to hyperadrenalism appear in Table XII. In both instances the anatomical diagnosis is based on gross and microscopic examination of the operative pathological specimen. It should be noted that there was no disturbance of blood acid-base balance in patient $\mathrm{J}$. T. at the time of study. Although $\mathrm{CO}_{2}$ retention was documented in this patient only for the two-month period of observation prior to therapy, clinical symptoms had been present for a year or more. The venous serum $\mathrm{CO}_{2}$ content, previously about $35 \mathrm{mEq}$. per liter, fell to normal shortly after subtotal adrenalectomy, and remained so during the ensuing four months prior to study.

The spirometric observations on these two patients are shown in Table XII. The slight reductions in vital and maximum breathing capacities found in patient $\mathrm{K}$. S. were probably the result of old pleural disease.
Tables XIII, XIV, and XV contain the data obtained during $\mathrm{CO}_{2}$ inhalation in these two patients. The first was found to have an elevated arterial blood $\mathrm{pH}$ and serum bicarbonate level at rest, with a somewhat lowered oxygen saturation. Resting values for the second patient were within normal limits at the time of study. In both cases the ventilatory responses to 3 per cent and 5 per cent $\mathrm{CO}_{2}$ inhalation were less than normal, and sensitivity to the carbon dioxide-hydrogen ion stimulus was one-half to one-third that represented by the average normal values.

\section{DISCUSSION}

The effects of $\mathrm{CO}_{2}$ inhalation on the normal subjects were comparable to those found previously (21-25). In this study the average value of 1.7 mm. $\mathrm{Hg}$ change in arterial $\mathrm{CO}_{2}$ tension required

TABLE XV

Physiologic data relative to effective alveolar ventilation and sensitivity to the carbon dioxide-hydrogen ion stimulus in patients with cyanotic congenital heart disease, chronic acidosis, and chronic alkalosis

\begin{tabular}{|c|c|c|c|c|c|c|c|c|}
\hline Patient & $\begin{array}{c}\text { Inspired } \\
\text { gas } \\
\text { mixture }\end{array}$ & $\dot{\mathbf{v}}_{\mathbf{A}}$ & $\dot{\mathbf{V}}_{\mathbf{A}} / \mathbf{M}^{\mathbf{2}}$ & VR & $\frac{\mathrm{Pa}_{\mathrm{CO}_{2}}}{\mathrm{VR}}$ & $\frac{\hat{V}_{\mathbf{A}} / \mathbf{M}_{\mathbf{2}}}{\mathrm{Pa}_{\mathrm{CO}_{2}}}$ & $\frac{\left(\mathrm{H}^{+}\right) \mathbf{a}}{\mathrm{VR}}$ & $\frac{\dot{V}_{\mathbf{L}} / \mathbf{M}^{\mathbf{2}}}{\left(\mathbf{H}^{+}\right)_{\mathbf{a}}}$ \\
\hline \multicolumn{9}{|c|}{ Cyanotic congenital heart disease } \\
\hline J. B. & $\begin{array}{l}\text { Room air } \\
3 \% \mathrm{CO}_{2} \\
4 \% \mathrm{CO}_{2}\end{array}$ & $\begin{array}{l}5.63 \\
21.80 \\
40.7\end{array}$ & $\begin{array}{r}3.37 \\
13.05 \\
24.36\end{array}$ & $\begin{array}{l}1 \\
3.88 \\
7.22\end{array}$ & 1.0 & 3.5 & 0.7 & 5.5 \\
\hline R.W. & $\begin{array}{l}\text { Room air } \\
3 \% \mathrm{CO}_{2} \\
4 \% \mathrm{CO}_{2}\end{array}$ & $\begin{array}{r}5.08 \\
8.12 \\
15.68\end{array}$ & $\begin{array}{l}3.10 \\
4.95 \\
9.56\end{array}$ & $\begin{array}{l}1 \\
1.60 \\
3.08\end{array}$ & 2.2 & 1.5 & 1.8 & 1.7 \\
\hline W. M. & $\begin{array}{l}\text { Room air } \\
3 \% \mathrm{CO}_{2} \\
4 \% \mathrm{CO}_{2}\end{array}$ & $\begin{array}{r}6.54 \\
15.33 \\
25.80\end{array}$ & $\begin{array}{r}3.53 \\
8.24 \\
13.87\end{array}$ & $\begin{array}{l}1 \\
2.34 \\
3.94\end{array}$ & 2.7 & 1.3 & 2.6 & 1.4 \\
\hline \multicolumn{9}{|c|}{ Chronic acidosis } \\
\hline G. $\mathbf{K}$. & $\begin{array}{l}\text { Room air } \\
3 \% \mathrm{CO}_{2} \\
5 \% \mathrm{CO}_{2}\end{array}$ & $\begin{array}{r}3.96 \\
9.16 \\
19.75\end{array}$ & $\begin{array}{r}2.50 \\
5.80 \\
12.50\end{array}$ & $\begin{array}{l}1 \\
2.32 \\
5 .\end{array}$ & 1.4 & 1.7 & 1.4 & 1.6 \\
\hline J. K. & $\begin{array}{l}\text { Room air } \\
3 \% \mathrm{CO}_{2} \\
5 \% \mathrm{CO}_{2}\end{array}$ & $\begin{array}{c}5.22 \\
12.14 \\
-\end{array}$ & $\begin{array}{l}3.13 \\
7.27 \\
-\end{array}$ & $\stackrel{1}{2.33}$ & 0.4 & 8.0 & 0.7 & 4.8 \\
\hline W. E. & $\begin{array}{l}\text { Room air } \\
3 \% \mathrm{CO}_{2} \\
5 \% \mathrm{CO}_{2}\end{array}$ & $\begin{array}{r}4.74 \\
10.98 \\
-\end{array}$ & $\begin{array}{l}2.48 \\
5.75 \\
-\end{array}$ & $\begin{array}{l}1 \\
2.31 \\
-\end{array}$ & 2.0 & 1.4 & 1.0 & 2.0 \\
\hline \multicolumn{9}{|c|}{ Chronic alkalosis } \\
\hline K.S. & $\begin{array}{l}\text { Room air } \\
3 \% \mathrm{CO}_{2} \\
5 \% \mathrm{CO}_{2}\end{array}$ & $\begin{array}{l}3.63 \\
6.03 \\
9.78\end{array}$ & $\begin{array}{l}2.20 \\
3.65 \\
5.93\end{array}$ & $\begin{array}{l}1 \\
1.66 \\
2.69\end{array}$ & 4.3 & 0.5 & 2.5 & 0.9 \\
\hline J. T. & $\begin{array}{l}\text { Room air } \\
3 \% \mathrm{CO}_{2} \\
5 \% \mathrm{CO}_{2}\end{array}$ & $\begin{array}{l}3.46 \\
5.51 \\
8.70\end{array}$ & $\begin{array}{l}2.18 \\
3.46 \\
5.47\end{array}$ & $\begin{array}{l}1 \\
1.59 \\
2.51\end{array}$ & 6.6 & 0.3 & 3.2 & 0.7 \\
\hline
\end{tabular}


to double the effective alveolar ventilation in normal subjects is not much different from the value of $1.5 \mathrm{~mm}$. $\mathrm{Hg}$ obtained by Haldane and Priestley (21) as early as 1905 , using alveolar air samples. Gray (17) arrived at an average figure of $2.5 \mathrm{~mm}$. $\mathrm{Hg}$ using 200 determinations reported in the literature under both steady and unsteady conditions, and based on alveolar air $\mathrm{CO}_{2}$ tensions. The buffering capacity of normal human blood, measured in vivo under conditions of $\mathrm{CO}_{2}$ inhalation and voluntary hyperventilation, has previously been reported by Shock and Hastings (26). It was found that when arterial hydrogen ion concentration is plotted against arterial $\mathrm{CO}_{2}$ tension in $\mathrm{mm}$. $\mathrm{Hg}$, an almost linear relationship is obtained which is approximated in the hypercapnial range by the equation $\left(\mathrm{H}^{+}\right) \mathrm{a}=0.652 \cdot \mathrm{PaCO}_{2}+13.5 \quad$ (17). When plotted in the same way, the data obtained in this study using normal subjects also yield a linear relationship, the equation for which is $\left(\mathrm{H}^{+}\right) \mathrm{a}=0.714 \mathrm{PaCO}_{2}+8.3$.

The increase in physiological dead space to $\mathrm{CO}_{2}$ (Bohr) accompanying the hyperpnea of $\mathrm{CO}_{2}$ inhalation in normal subjects has long been recognized (24), and the magnitude of change observed in this study is comparable to that previously reported. Changes of this order of magnitude in the respiratory dead space with comparable increases in tidal volume have not been found using the nitrogen analysis technique (27). However, the size of the Bohr dead space to $\mathrm{CO}_{2}$ is subject to changes in ventilation perfusion relationships in the lung, and may be increased, for example, when the lung contains appreciable numbers of well ventilated but poorly perfused alveoli. The increase in physiological dead space observed in this study with $\mathrm{CO}_{2}$ inhalation is interpreted as suggesting a change in ventilation perfusion relationships under these conditions, perhaps secondary to changes in alveolar volume, or in respiratory air flow velocity, or possibly resulting from an effect of increased $\mathrm{CO}_{2}$ tension on the pulmonary capillaries or bronchioles.

In evaluating the reduction in sensitivity to the carbon dioxide-hydrogen ion stimulus found in the Group III and IV emphysema patients, the possible effect of the hypoxic ventilatory drive at rest must be taken into consideration. As pointed out by Tenney (5), it might be expected that the diminution in anoxemia coincident with $\mathrm{CO}_{2}$ in- halation would reduce this drive, and therefore the observed slope of the stimulus response curve might be less. As noted above, in these patients there was little or no further rise in arterial oxygen saturation with 5 per cent $\mathrm{CO}_{2}$ inhalation as compared with 3 per cent $\mathrm{CO}_{2}$, so that there was presumably no change in the degree of hypoxic drive between these two points on the stimulus response curve. Yet the slope of the curves between these two points did not differ from that between those determined at rest and on 3 per cent $\mathrm{CO}_{2}$ inhalation, there being a consistent linear relationship of the three. Therefore changing hypoxic drive, such as may have occurred, did not modify the results obtained in any detectable way.

The reduction in sensitivity to the carbon dioxide-hydrogen ion stimulus found in patients with cor pulmonale secondary to emphysema might be ascribed to one or more of the following mechanisms: 1) Increased buffering capacity of the blood associated with an elevated plasma bicarbonate level or polycythemia ; 2 ) failure of the chest bellows to respond adequately to the normal nervous stimuli; 3 ) the presence of congestive heart failure, chronic anoxemia, chronic acidosis, or chronic hypercapnia per se.

In 1920, Scott (3) suggested that the reduced ventilatory response to $\mathrm{CO}_{2}$ inhalation which he observed in two patients with chronic emphysema might be explained on the basis of an increased buffering capacity of the blood secondary to a high bicarbonate level. This would result in a smaller increment in free hydrogen ion concentration on addition of a given amount of $\mathrm{CO}_{2}$, and therefore a lesser ventilatory response. However, our measurements show that with $\mathrm{CO}_{2}$ inhalation the blood hydrogen ion concentration in these patients actually increases more than in the normal group despite the presence of elevated blood bicarbonate and polycythemia. Thus the sensitivity is reduced as referred to either the $\mathrm{CO}_{2}$ or hydrogen ion stimulus. Furthermore the absence of a reduced sensitivity in the patients with marked polycythemia associated with cyanotic congenital heart disease would argue against a specific effect of polycythemia in this regard.

The presence of mechanical ventilatory impairment did not condition the diminished sensitivity since a normal response was found in the Group II emphysema patients who suffered from com- 
parable ventilatory defects. Likewise the presence of congestive heart failure per se does not account for the diminished sensitivity in cor pulmonale, since a reduction was also found in the Group III emphysema patients without congestive difficulty, and conversely one of the Group II emphysema patients with congestive failure on another basis had a normal response.

Since the anoxemia and acidosis of the patients with cor pulmonale were presumably of long duration, it appeared that a pertinent question in the present study was whether or not either chronic anoxemia or chronic acidosis might in themselves result in a diminished sensitivity to the carbon dioxide-hydrogen ion stimulus. Although the available evidence would indicate an increase in sensitivity to $\mathrm{CO}_{2}$ associated with acclimatization to the anoxemia of altitude $(22,28)$, these observations were made over a period of days or weeks, and it was considered desirable to investigate the possibility that anoxemia of considerably longer duration might result in a diminished sensitivity. Likewise, although the observations of Peabody (19) suggested that if anything, an increased sensitivity to $\mathrm{CO}_{2}$ was associated with renal failure, it seemed that the possible effects of known long-term acidosis should be explored. Accordingly, the patients with cyanotic congenital heart disease and slowly progressing uremia were selected to fulfill the criterion of chronicity. The absence of diminished sensitivity in either of these two patient categories has been taken as evidence that neither chronic anoxemia nor chronic acidosis in themselves condition the reduced response in patients with cor pulmonale.

The hypothesis that chronic hypercapnia itself might eventually lead to a diminished sensitivity to the carbon dioxide-hydrogen ion stimulus, although likely on the basis of exclusion, was more difficult of direct test. The hypercapnia of metabolic alkalosis seemed to offer the best clinical opportunity to explore this possibility, but long standing severe alkalosis and $\mathrm{CO}_{2}$ retention are infrequently seen. The two patients with Cushing's syndrome therefore were chosen because of well-documented evidence of chronic metabolic alkalosis and $\mathrm{CO}_{2}$ retention, there being no reason to believe that other metabolic derangements in these patients might condition the ventilatory response. As was the case with the emphysematous subjects, the diminution in sensitivity with reference to hydrogen ion precludes the possibility that increased buffering capacity of the blood resulted in a reduced response to $\mathrm{CO}_{2}$. The findings are therefore taken as evidence that chronic hypercapnia per se leads to a diminution in sensitivity to the carbon dioxide-hydrogen ion stimulus. This thesis is further supported by the findings of Schäfer (29), who observed a reduced response to $\mathrm{CO}_{2}$ in two normal men after four to six days residence in an atmosphere of 3 per cent $\mathrm{CO}_{2}$.

Since both respiratory acidosis and metabolic alkalosis are associated with elevations in blood and tissue bicarbonate ion concentration as well as free carbon dioxide tension, no conclusion can be drawn from these observations as to which of these two excess quantities may lead to a reduced sensitivity, or whether both together do.

The findings in this study lend no support to the idea $(30,31)$ that an increased ventilatory response to $\mathrm{CO}_{2}$ necessarily accompanies the condition of metabolic acidosis by reason of the lowered buffering capacity of the blood resulting in a greater increase in hydrogen ion concentration with the addition of a given amount of $\mathrm{CO}_{2}$. Firstly, no increase in response to $\mathrm{CO}_{2}$ was observed in two of the three patients with metabolic acidosis, and secondly, the patient demonstrating an increased response was found to have increased sensitivity in terms of hydrogen ion as well as $\mathrm{CO}_{2}$. This latter observation would be consistent with the notion that an increase in sensitivity may accompany chronic hypocapnia, since this patient was observed to have a lowered venous serum $\mathrm{CO}_{2}$ content on occasion for several years. On the other hand, if $\mathrm{CO}_{2}$ acts as a respiratory stimulus only by increasing the hydrogen ion concentration in blood and tissue, then it might be expected that chronic metabolic acidosis would result in some diminution in sensitivity to the carbon dioxidehydrogen ion stimulus as is observed with respiratory acidosis. The absence of such diminution with metabolic acidosis therefore may be construed as evidence favoring the theory that $\mathrm{CO}_{2}$ acts as a specific stimulus.

We have interpreted the data as consistent with the hypothesis of $\mathrm{CO}_{2}$ adaptation in prolonged hyper- and hypocapnia, and with the multiple factor theory of the chemical regulation of respiration advanced by Gray (17). 
Little information is available presently as regards the degree of reversibility possible once a chronic diminution in sensitivity has become established. It is noteworthy that a diminished sensitivity was found in patient $\mathrm{J}$. T. with Cushing's syndrome some four months after the venous serum $\mathrm{CO}_{2}$ content had returned to normal.

The sequence of events in the natural history of chronic pulmonary emphysema leading to $\mathrm{CO}_{2}$ retention is at present poorly defined. A tentative hypothesis suggested by these studies is the following: At some point in the course of the disease the ventilatory capacity becomes insufficient to eliminate the additional $\mathrm{CO}_{2}$ produced during exertion, and transient rises in arterial $\mathrm{CO}_{2}$ tension occur. Eventually these repeated bouts of hypercapnia result in a diminished sensitivity to the carbon dioxide-hydrogen ion stimulus, thus permitting an elevated $\mathrm{CO}_{2}$ tension at rest though ventilatory capacity may be such that a normal $\mathrm{CO}_{2}$ tension at an increased resting ventilation is possible. Furthermore, this elevated $\mathrm{CO}_{2}$ tension at rest favors increased retention of bicarbonate by the kidney $(32,33)$ and thus a vicious cycle of increasing $\mathrm{CO}_{2}$ retention and diminishing respiratory sensitivity is initiated.

The question of whether increasing $\mathrm{CO}_{2}$ retention is associated with further diminution in sensitivity to the carbon dioxide-hydrogen ion stimu- lus cannot be answered with certainty at this time. Presumably serial observations on a number of patients would be needed. Lacking these, we have attempted to correlate the degree of sensitivity change with the amount of $\mathrm{CO}_{2}$ retention in those patients having a reduced response to the carbon dioxide-hydrogen ion stimulus. In Figures 3 and 4 sensitivity is expressed both as the change in effective alveolar ventilation per square meter body surface area associated with $1 \mathrm{~mm}$. $\mathrm{Hg}$ increase in arterial $\mathrm{CO}_{2}$ tension, and as the change associated with unit increase in arterial hydrogen ion concentration. Sensitivity is plotted against the level of arterial $\mathrm{CO}_{2}$ tension at rest. It is apparent that the sensitivity tends to diminish as the resting arterial $\mathrm{CO}_{2}$ tension increases. No correlation between sensitivity and arterial serum $\mathrm{CO}_{2}$ content in these same patients is demonstrable.

It is difficult to define possible therapeutic implications of these observations at this time. Nevertheless, it would seem reasonable to suppose that circumstances making for further $\mathrm{CO}_{2}$ retention in some patients, such as acute pulmonary infection, vigorous exertion, or long continued oxygen therapy, would further accelerate a vicious cycle of diminishing sensitivity to $\mathrm{CO}_{2}$, with resultant reduction in effective alveolar ventilation at rest, increased anoxemia and acidosis.

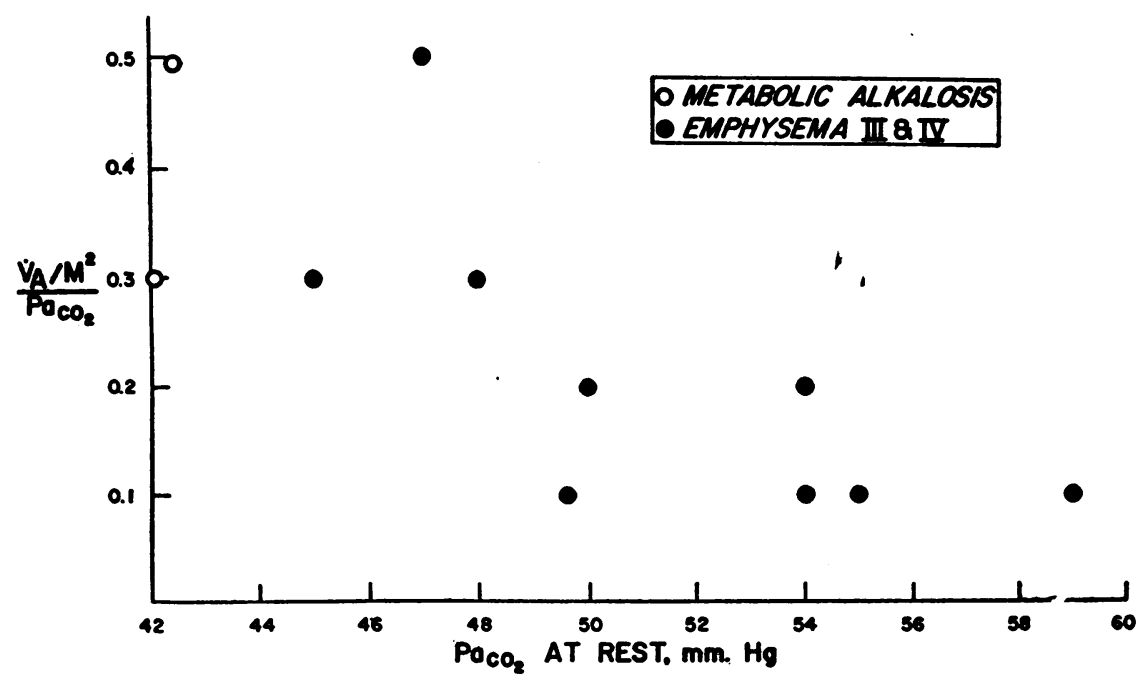

Fig, 3. Senstivity to the $\mathrm{CO}_{2}$ Stmulus as a Function of the Degree of Carbon Dioxide Retention

Sensitivity appears on the ordinate in terms of increase in effective alveolar ventilation (liters BTPS per square meter body surface area) associated with $1 \mathrm{~mm}$. $\mathbf{H g}$ rise in arterial $\mathrm{CO}_{2}$ tension. On the abscissa is plotted arterial $\mathrm{CO}_{2}$ tension at rest. 


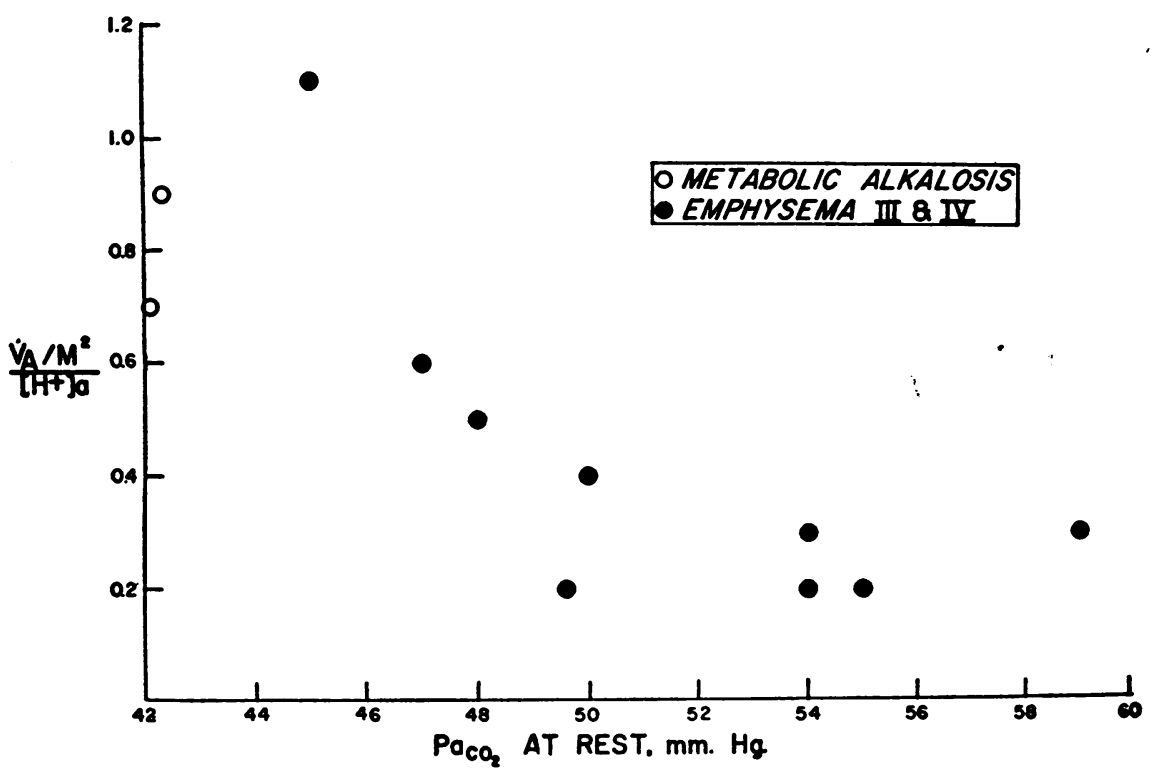

Fig. 4. Sensitivity to the Hydrogen Ion Stimulus as a Function of the Degree of Carbon Dioxide Retention

Sensitivity appears on the ordinate in terms of increase in effective alveolar ventilation (liters BTPS per square meter body surface area) associated with unit rise in arterial hydrogen ion concentration (billionths of moles per liter). On the abscissa is plotted arterial $\mathrm{CO}_{2}$ tension at rest.

\section{SUMMARY AND CONCLUSIONS}

1. In 12 normal subjects and 21 patients, sensitivity of the respiratory nervous regulatory mechanism to the carbon dioxide-hydrogen ion stimulus has been determined by relating effective alveolar ventilation to changes in arterial $\mathrm{CO}_{2}$ tension and hydrogen ion concentration induced by $\mathrm{CO}_{2}$ inhalation.

2. Six patients with chronic pulmonary emphysema and cor pulmonale were found to have a markedly reduced sensitivity to the carbon dioxidehydrogen ion stimulus as compared with the normal.

3. Several mechanisms of possible importance in conditioning the reduced ventilatory response to $\mathrm{CO}_{2}$ in the patients with cor pulmonale have been explored. They are: (a) increased buffering capacity of the blood associated with an elevated plasma bicarbonate level or polycythemia; (b) failure of the chest bellows to respond adequately to the normal nervous stimuli; (c) the presence of congestive heart failure, chronic anoxemia, chronic acidosis, or chronic hypercapnia per se.

4. The diminished response to $\mathrm{CO}_{2}$ in the patients with cor pulmonale could not be accounted for on the basis of increased blood buffering ca- pacity since the sensitivity was found to be reduced relative to the hydrogen ion as well as to the $\mathrm{CO}_{2}$ stimulus.

5. Four patients without $\mathrm{CO}_{2}$ retention but having chronic pulmonary emphysema and a mechanical ventilatory defect similar to that of the cor pulmonale group were found to have normal sensitivity to the carbon dioxide-hydrogen ion stimulus.

6. The diminished sensitivity in patients with cor pulmonale was not dependent upon the presence of congestive heart failure since three patients without congestive difficulty but having emphysema and $\mathrm{CO}_{2}$ retention were found to have a reduced sensitivity.

7. No reduction in sensitivity was found in three patients with chronic anoxemia secondary to cyanotic congenital heart disease, nor in three patients with chronic metabolic acidosis associated with renal failure.

8. In two patients with chronic metabolic alkalosis and $\mathrm{CO}_{2}$ retention, a diminished sensitivity was demonstrated.

9. It is concluded that chronic hypercapnia per $s e$ results in a diminished sensitivity to the $\mathrm{CO}_{2}$ inhalation stimulus, which is associated with a rise 
in both arterial $\mathrm{CO}_{2}$ tension and hydrogen ion concentration.

10. Implications of these results are discussed in terms of the specificity of $\mathrm{CO}_{2}$ as a respiratory stimulus and the development of reduced sensitivity in emphysematous subjects.

\section{REFERENCES}

1. Baldwin, E. deF., Cournand, A., and Richards, D. W., Jr., Pulmonary insufficiency. III. A study of 122 cases of chronic pulmonary emphysema. Medicine, 1949, 28, 201.

2. Reinhardt, R., Uber das Verhältnis von $\mathrm{CO}_{2}$-Ausscheidung zur Atemgrösse beim Lungenemphysem. Deut. Arch. f. Klin. Med., 1912, 109, 192.

3. Scott, R. W., Observations on the pathologic physiology of chronic pulmonary emphysema. Arch. Int. Med., 1920, 26, 544.

4. Donald, K. W., and Christie, R. V., The respiratory response to carbon dioxide and anoxia in emphysema. Clin. Sc., 1949, 8, 33.

5. Tenney, S. M., Ventilatory response to carbon dioxide in pulmonary emphysema. J. Applied Physiol., 1954, 6, 477.

6. Baldwin, E. deF., Cournand, A., and Richards, D. W., Jr., Pulmonary insufficiency. I. Physiological classification, clinical methods of analysis, standard values in normal subjects. Medicine, 1948, 27, 243.

7. Nielsen, M., Untersuchungen über die Atemregulation beim Menschen, besonders mit Hinblick auf die Art des chemischen Reizes. Skandinav. Arch. f. Physiol., Suppl. 10, 87, 1936.

8. Bohr, C., Uber die Lungenathmung. Skandinav. Arch. f. Physiol., 1891, 2, 236.

9. Bock, A. V., Dill, D. B., Edwards, H. T., Henderson, L. J., and Talbott, J. H., On partial pressures of oxygen and carbon dioxide in arterial blood and alveolar air. J. Physiol., 1929, 68, 277.

10. Riley, R. L., Lilienthal, J. L., Jr., Proemmel, D. D., and Franke, R. E., On the determination of the physiologically effective pressures of oxygen and carbon dioxide in alveolar air. Am. J. Physiol., 1946, 147, 191.

11. Gray, J. S., Concerning the use of $\mathrm{CO}_{2}$ to counteract anoxia. Proj. No. 310, AAF School of Aviation Med., Randolph Field, Texas, 26 Aug., 1944.

12. Fenn, W. O., Rahn, H., and Otis, A. B., A theoretical study of the composition of the alveolar air at altitude. Am. J. Physiol., 1946, 146, 637.

13. Singer, R. B., and Hastings, A. B., An improved clinical method for the estimation of disturbances of the acid-base balance of human blood. Medicine, 1948, 27, 223.

14. Standardization of definitions and symbols in respiratory physiology. Federation Proc., 1950, 9, 602.
15. Scholander, P. F., Analyzer for accurate estimation of respiratory gases in one-half cubic centimeter samples. J. Biol. Chem., 1947, 167, 235.

16. Darling, R. C., Cournand, A., and Richards, D. W., Jr., Studies on the intra-pulmonary mixture of gases. III. An open circuit method for measuring residual air. J. Clin. Invest., 1940, 19, 609.

17. Gray, J. S., Pulmonary ventilation and its physiological regulation. American Lecture Series, Publication No. 63, Springfield, Charles C Thomas, 1950.

18. Robinson, S., Experimental studies of physical fitness in relation to age. Arbeitsphysiologie, 1938, 10, 251.

19. Peabody, F. W., Clinical studies on the respiration. I. The effect of carbon dioxide in the inspired air on patients with cardiac disease. Arch. Int. Med., $1915,16,846$.

20. Yeomans, A., and Stueck, G. H., Jr., Clinical-chemical studies of acid-base abnormalities. Changes in acid-base balance observed in renal and respiratory disease. Am. J. Med., 1952, 13, 183.

21. Haldane, J. S., and Priestley, J. G., The regulation of the lung-ventilation. J. Physiol., 1905, 32, 225.

22. Hasselbalch, K. A., and Lindhard, J., Analyse des Höhenklimas in Seinen Wirkungen auf die Respiration. Skandinav. Arch. f. Physiol., 1911, 25, 361.

23. Lindhard, J., On the excitability of the respiratory centre. J. Physiol., 1911, 42, 337.

24. Campbell, J. M. H., Douglas, C. G., and Hobson, F. G., The sensitiveness of the respiratory centre to carbonic acid, and the dead space during hyperpnoea. J. Physiol., 1914, 48, 303.

25. Davies, H. W., Brow, G. R., and Binger, C. A. L., The respiratory response to carbon dioxide. $\mathrm{J}$. Exper. Med., 1925, 41, 37.

26. Shock, N. W., and Hastings, A. B., Studies of the acid-base balance of the blood. IV. Characterization and interpretation of displacement of the acid-base balance. J. Biol. Chem., 1935, 112, 239.

27. Fowler, W. S., Lung function studies II. The respiratory dead space. Am. J. Physiol., 1948, 154, 405.

28. Rahn, H., and Otis, A. B., Man's respiratory response during and after acclimatization to high altitude. Am. J. Physiol., 1949, 157, 445.

29. Schäfer, K.-E., Atmung und Säure-Basengleichgewicht bei langdauerdem Aufenthalt in $3 \% \mathrm{CO}_{2}$. Pflüger's Arch. f. d. ges. Physiol., 1949, 251, 689.

30. Gesell, R., Respiration and its adjustments. Ann. Rev. Physiol., 1939, 1, 185.

31. Hesser, C. M., Central and chemoreflex components in the respiratory activity during acid-base displacements in the blood. Acta physiol. Scandinav., vol. 18, suppl. 64, 1949.

32. Brazeau, P., and Gilman, A., Effect of plasma $\mathrm{CO}_{2}$ tension on renal tubular reabsorption of bicarbonate. Am. J. Physiol., 1953, 175, 33.

33. Dorman, P. J., Sullivan, W. J., and Pitts, R. F., The renal response to acute respiratory acidosis. J. Clin. Invest., 1954, 33, 82. 